\title{
Advanced Stochastic Optimization Algorithm for Deep Learning Artificial Neural Networks in Banking and Finance Industries
}

\author{
Jamilu Auwalu Adamu ${ }^{1}$ \\ ${ }^{1}$ Mathematics Programme, 118 National Mathematical Centre, FCT-Abuja, Nigeria \\ Correspondence: Jamilu Auwalu Adamu, Mathematics Programme Building, 118 National Mathematical Centre, \\ Small Sheda, Kwali, FCT-Abuja, Nigeria. Tel: 234-803-867-9094. E-mail: whitehorseconsult@yahoo.com
}

Received: October 22, 2019 Accepted: November 20, 2019 Online Published: November 26, 2019

\begin{abstract}
One of the objectives of this paper is to incorporate fat-tail effects into, for instance, Sigmoid in order to introduce Transparency and Stability into the existing stochastic Activation Functions. Secondly, according to the available literature reviewed, the existing set of Activation Functions were introduced into the Deep learning Artificial Neural Network through the "Window" not properly through the "Legitimate Door" since they are "Trial and Error "and "Arbitrary Assumptions", thus, the Author proposed a "Scientific Facts", "Definite Rules: Jameel's Stochastic ANNAF Criterion", and a "Lemma" to substitute not necessarily replace the existing set of stochastic Activation Functions, for instance, the Sigmoid among others. This research is expected to open the "Black-Box" of Deep Learning Artificial Neural networks. The author proposed a new set of advanced optimized fat-tailed Stochastic Activation Functions EMANATED from the AI-ML-Purified Stocks Data namely; the Log - Logistic (3P) Probability Distribution (1st), Cauchy Probability Distribution (2nd), Pearson 5 (3P) Probability Distribution (3rd), Burr (4P) Probability Distribution (4th), Fatigue Life (3P) Probability Distribution (5th), Inv. Gaussian (3P) Probability Distribution (6th), Dagum (4P) Probability Distribution (7th), and Lognormal (3P) Probability Distribution (8th) for the successful conduct of both Forward and Backward Propagations of Deep Learning Artificial Neural Network. However, this paper did not check the Monotone Differentiability of the proposed distributions. Appendix A, B, and C presented and tested the performances of the stressed Sigmoid and the Optimized Activation Functions using Stocks Data (2014-1991) of Microsoft Corporation (MSFT), Exxon Mobil (XOM), Chevron Corporation (CVX), Honda Motor Corporation (HMC), General Electric (GE), and U.S. Fundamental Macroeconomic Parameters, the results were found fascinating. Thus, guarantee, the first three distributions are excellent Activation Functions to successfully conduct any Stock Deep Learning Artificial Neural Network. Distributions Number 4 to 8 are also good Advanced Optimized Activation Functions. Generally, this research revealed that the Advanced Optimized Activation Functions satisfied Jameel's ANNAF Stochastic Criterion depends on the Referenced Purified AI Data Set, Time Change and Area of Application which is against the existing "Trial and Error "and "Arbitrary Assumptions" of Sigmoid, Tanh, Softmax, ReLu, and Leaky ReLu.
\end{abstract}

Keywords: activation functions, probability distribution, Fat-tail, Jameel's ANNAF Stochastic Criterion, stocks, Referenced AI-Data Set

\section{Introduction}

Casper Hansen (2019) says "Better optimized neural network; choose the right activation function, and your neural network can perform vastly better".

Artist Hans Hoffman wrote, "The ability to simplify means to eliminate the unnecessary so that the necessary may speak."

On Tuesday, June 25, 2019, the U.S. Subcommittee on Communications, Technology, Innovation, and the Internet headed by Sen. John Thune, R-S.D. convened a hearing entitled, "Optimizing for Engagement: Understanding the Use of Persuasive Technology on Internet Platforms", to find appropriate policy on algorithms' transparency or explanation as regards to its decision-making and machine learning on internet platforms might be influencing the public. Some of the issues discussed includes; the AI Algorithms' transparency, regulations, supervision, secrecy, the Black Box, difficulty in terms of explainability, human bias, and final ranking, the algorithms bad recommendations, AI was accused will be used to determine the next US President and exposes children to unsolicited sexual videos contents. 
Thanks to the idea of Artificial Intelligence and Machine Learning, it makes approval of Loans and opening accounts automated using MyBucks, OnDeck, Kabbage, Lend up, Knab and Knab Finance, however, this has significantly reduced customers' time wait for the processing of loan request to just a few seconds. Presently, Artificial Intelligence has become an integral part of the Banking and Finance applications. The path to model deployment in banking and finance is traditionally cumbersome. Deploying a model is hard and takes time, resources and coordination across many teams throughout the bank.

In Bank and Finance Industries, one of giant and bravery first steps was take in 2015 by Ally Bank (USA) thereby introduced Ally Assist: "a chatbot that could respond to voice and text, make payments on behalf of the customer, give an account summary, monitor savings, spending patterns, and use natural language processing to understand and address customer queries". Banks around the globe launched best versions of chatbots: Erica to iPAL, Eva and the most famous one, SBI's SIA. The banking and finance sectors have witnessed momentums development and continue to increase their spending on artificial intelligence and ML. The years 2016 and 2017 marked a significant milestone in the development of AI due to AlphaGo remarkable games successes. PwC (2017) stated that global spending in artificial intelligence in Banking has reached \$5.1 billion. The IHS Markit's stated that the report claims it will grow up to $\$ 41.1$ billion in 2018 and expected to reach $\$ 300$ billion by 2030 .

The basic challenge is how to explore an Optimized ways where artificial intelligence and machine learning algorithms can be harnessed to be able to capture fat-tailed effects for accurate future prediction so as to improve Credit Decisions, Risk Assessment and Management, Fraud Prevention, Customer Experiences and apply to the other fields.

The key question you will be hearing in the Banking and Finance Industries is" What is Next? What comes Next? Where is ROI". Artificial intelligence and machine learning are said to revolutionize the financial world, changing the banking and finance experience for the better. However, there is an inherent risk of relying on AI and ML models. With a company's reputation, regulatory requirements, and finances at stake, it's critical to understand how to continuously monitor and validate the AI and ML models to ensure proper performance and sound business decisions. However, due to an increased reliance on models for everyday business processes and decisions, model risk must be effectively managed. If left unchecked, the consequences of model risk can be severe; where model risk is defined as the risk of financial or reputation loss due to errors in the development, implementation or use of models. Therefore, AI and machine learning models require constant monitoring and effective validation. This is not only a regulatory requirement, but it is also sound business practice.

Recent technological advancements have accelerated the integration of AI and machine learning models into more and more banking processes. In today's banking industry, institutions not using AI and machine learning risk losing their competitive edge, as competitors are increasingly enhancing their strategic decisions with the powerful analytical capabilities of AI and machine learning.

The contemporary Extremistan World (the statistical properties rapidly change over time), non-Gaussian (often skewed and exhibiting fat tails, making extreme events far more likely than they normally would be) where some things are inherently unpredictable even with Artificial Intelligence (Nassim N. Taleb (2013)). Couple with an inappropriate choice of activation functions.

A model may be reasonable, but the world itself may be unstable. "A good model today may be inappropriate tomorrow" (Emanuel Derman, 1996). 'Change' is in the timeframe of the beholder. Ben Steiner (2019) stated that the challenges of Deep Learning are basically, the Non-Stationarity challenge which is one problem with many names, namely; Concept Drift, Covariate Shift or DATASET SHIFT. He defined Dataset Drift as when the statistical properties of the target variable, which the model is trying to predict, change over time in unforeseen ways. The unforeseen substitution of one data source $\mathbf{S} 1$ (with underlying probability distribution $\mathbf{\Pi S 1}$ ), with another source $\mathbf{S} 2$ (with distribution $\mathbf{\Pi} \mathbf{S} 2$ ) imposes the Risk model. The second challenge was that we are learning what we already knew, Learning, but nothing new is incorporate into the learning process to capture the effects of unforeseen challenges, basically, the LOW-PROBABILITY, HIGH-IMPACT (fat-tailed effects).

According to the available literature review, the existing set of Activation Functions were introduced into the Deep learning Artificial Neural Network through the "WINDOW" not properly through the "LEGITIMATE DOOR" since they are "TRIAL and ERROR "and "ARBITRARY ASSUMPTIONS". The objectives of this paper are to incorporate fat-tail effects into the existing stochastic Activation Functions, for instance, Sigmoid and proposed a Stochastic "DEFINITE RULES" and "LEMMA" EMANATED from the AI-ML-Data Set to substitute not necessarily replace the existing set of "Trial and Error" stochastic Activation Functions. This research is expected to open the "Black-Box" of Deep Learning Artificial Neural networks. 
The paper started with the Introduction, Literature Review, Material and Methods, Results; the paper crown up with Conclusion remarks.

\section{Literature Review}

Activation functions add a non-linearity to neural networks, thus gives it ability to learn complex functional mappings from data. A neural network without an activation function: (1) Can only represent a linear relationship between variables and, (2) Does not hold Universal approximation theorem. This can be seen below:

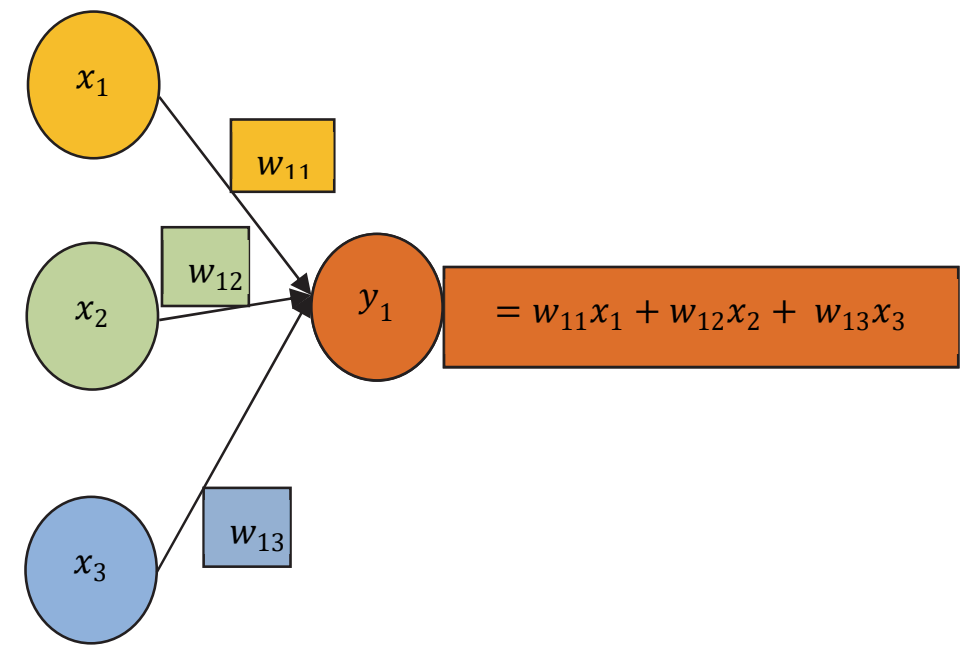

That means

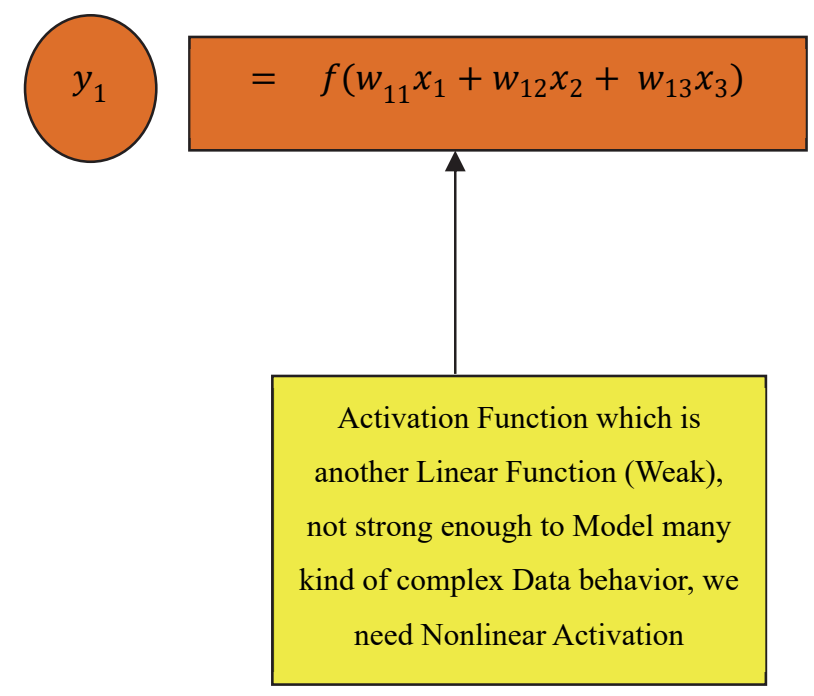

Figure 1. Linearity of Activation Functions

Source: Author (2019)

The choice of a Neural Network Activation Functions does not follow DEFINITE RULES; however, this makes the Deep Learning Artificial Neural Network a "Black Box" System and exposes its predictions to a "Model Risk".

Sebastian Urban (2017) stated that the choice of activation functions was not seriously challenged by researchers (except for special purpose applications), until recently when Nair et al. (2010) introduced the rectified linear unit (ReLU), a neuron with an activation function that is linear for positive inputs and zero for negative inputs.

This achievement led to a wave of follow-up research in activation functions specifically tailored to deep networks. The year 2015, introduced stochasticity into the activation function by sampling the value for the slope with each training iteration from a fixed uniform distribution. Clevert et al. (2015) and Klambauer et al. (2017) replaced the negative part of ReLUs with a scaled exponential function and showed that, under certain conditions, this leads to automatic renormalization of the inputs to the following layer and thereby simplifies the training of the neural networks, leading to accuracy improvements of deep feed-forward networks on tasks from the UCI Machine 
Learning repository (Lichman, 2013) amongst others. Nearly fully adaptable activation functions have been proposed by Eisenach et al. (2017). Similarly, Scardapane et al. (2017) also use a basis expansion, but with a set of Gaussian kernels that are equally distributed over a preset input range.

According to Asman Dureja and Payal Pahwa (2019), the choice of activation functions affects the neural network in term of OPTIMIZATION and to retrieve the better results. Several activation functions have been introduced in machine learning for many practical applications. But which activation function should use at hidden layer of deep neural networks was not identified. According to Chigozie Enyinna Nwankpa et al. (2018), LRELU, PReLU and RReLU perform better than the ReLU but some of these functions lack theoretical justifications to support their state-of-the-art results. Soufiane Hayou et al. (2019) stated that an inappropriate selection of Activation Function can lead to the loss of information of the input during forward propagation and the exponential vanishing/exploding of gradients during back-propagation. Schoenholz et al. (2017) applied Gaussian process approximation to Deep Neural Networks.

Joonho Lee et al. (2019), in their work, proposed a probabilistic activation function, called ProbAct. Unfortunately, the output value of ProbAct is sampled from a normal distribution; with the mean value same as the output of ReLU and with a fixed or trainable variance for each element.

Professionally, the opinions of the Members of StackExchange (2018) sponsored by IBM stated that "I choose the activation function for the output layer depending on the output that I need and the properties of the activation function that I know. In hidden layers, I use a leaky ReLU to avoid dead neurons instead of the ReLU and the tanh instead of the sigmoid. Of course, I don't use a linear function in hidden units. However, the choice for them in the hidden layer is mostly due to trial and error. However, the more activation functions I discover the more I'm confused about the choice of the function to use in hidden layers. And I don't think that flipping a coin is a good way of choosing an activation function. It would be incredibly difficult to recommend an activation function that works for all use cases (although I believe that SELU was designed so that it would do the right thing with pretty much any input).At the end of the day, you are probably going to get as many opinions as there are people about the right choice of activation function. The bottom line is that there is no universal rule for choosing an activation function for hidden layers".

\section{Materials and Methods}

\subsection{Materials}

\subsubsection{Basic Definitions}

\subsubsection{Artificial intelligence (AI)}

Artificial intelligence (AI) was created as a subfield of computer science targeted to solve tasks that humans are good at or the goal of AI is to mimic human intelligence. Artificial intelligence and machine learning technology in banking industry can be used for Credit decisions (It can allow for a fast, accurate assessment of a potential borrower, for far less cost than traditional methods, it eliminates bias, as machines have more objectivity than human employees), Risk assessment and management (banks mitigate risk because they receive accurate reporting, not prone to human error, AI does even more to reduce risks for banks and customers). By viewing the history of risk cases, AI can help banks forecast issues and take early steps to avoid problems), Fraud prevention (By analyzing spending patterns, location, and client behavior, machine learning can detect anomalies in spending and alert the cardholder, dramatically reducing credit card fraud) and Personalized approach (notifies members if their card was charged twice for an expense or if they tipped an exorbitant amount at a restaurant. 


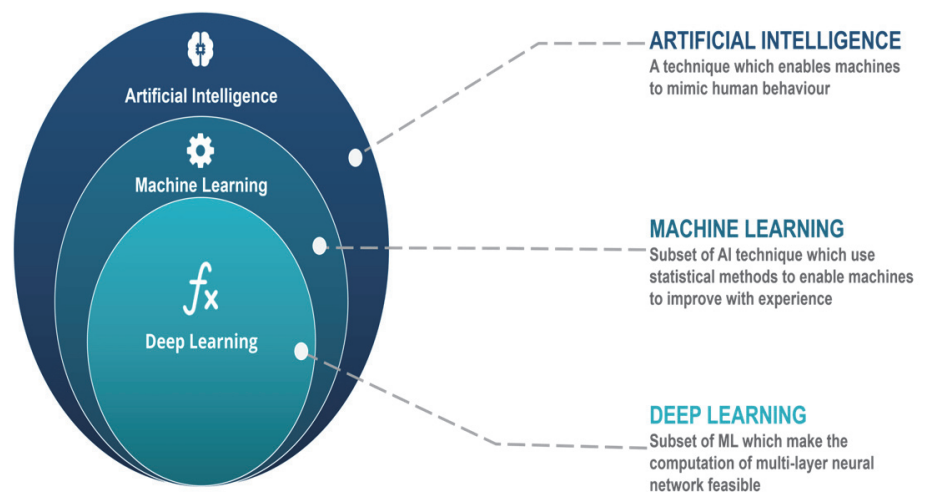

Figure 2. Artificial Intelligence

Source: Google Images (2019)

\subsubsection{Machine Learning}

Machine Learning uses algorithms to learn from data without relying on rules-based programming. Machine Learning originally developed as a subfield of Artificial Intelligence (AI), one of the goals behind machine learning was to replace the need for developing computer programs "manually." Considering that programs are being developed to automate processes, we can think of machine learning as the process of "automating automation." In other words, machine learning lets computers "create" programs (often, the intent for developing these programs is making predictions) themselves. In other words, machine learning is the process of turning data into programs. Machine learning (and deep learning) definitely helps to develop "AI," however, AI doesn't necessarily have to be developed using machine learning - although, machine learning makes "AI" much more convenient.

Machine learning models can also predict which banking tools individual members might use and recommend them so customers can make better financial decisions. Process automation (Using robotic process automation (RPA), banks can remove human error and restructure the workforce to focus on more pressing tasks. JPMorgan Chase \& Co launched COIN or Contract Intelligence, which automated the processing of legal documents, extraction of data, and review of certain types of legal contracts. Machine learning algorithms could use image recognition to identify patterns in the agreements. What normally would take roughly 360,000 labor hours per year, took the model a few hours. Another example of automating tasks is the increased use of chatbots that provide quick and reliable answers to consumers. Using AI-powered mobile and web chatbots, banks can speed up the time it takes for consumers to receive answers and decrease the need for human assistants to answer questions. Other applications are improved customer services, Investment Predictions Marketing, Network Security and Algorithm Trading.

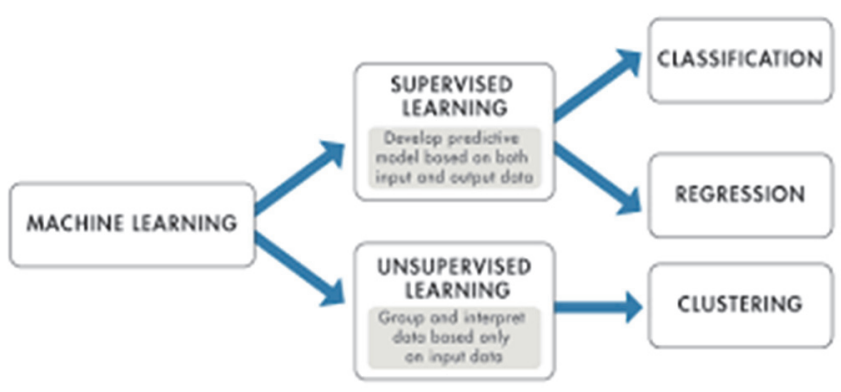

Figure 3. Machine Learning

Source: Google Images (2019) 


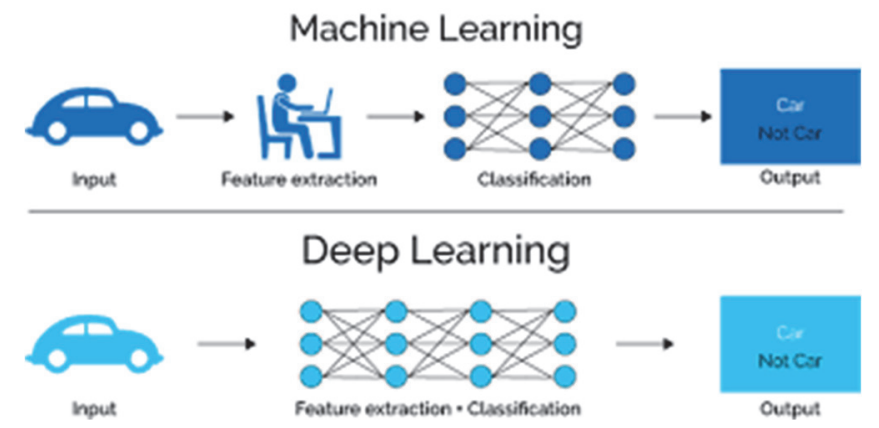

Figure 4. Difference between Machine Learning and Deep Learning

Source: Google Images (2019)

\subsubsection{Deep Learning}

Deep learning is a subfield of machine learning, referring to a particular subset of models that are particularly good at certain tasks. Deep Learning algorithms run data through several "layers" of neural network algorithms, each of which passes a simplified representation of the data to the next layer. The ability to process large numbers of features makes Deep Learning algorithms very powerful when dealing with unstructured data (such as images, audio and video). However, Deep Learning algorithms can be overkill for less complex problems because they require access to a vast amount of data to be effective and may be outperformed by Tree-Based Algorithms in these cases. Instead of organizing data to run through predefined equations, Deep Learning algorithms characterize basic parameters about the data and train the computer to learn on its own by recognizing patterns using many layers of processing. For instance, Deep Learning algorithms include: Recurrent Neural Networks (RNN), Deep Boltzmann Machines (DBM), Deep Belief Networks (DBN), Convolutional Neural Networks (CNN), and Stacked Auto-Encoders.

Deep Learning maps inputs to outputs using multiple layers of nonlinear processing units. It is capable of creating models for previously manual procedures. The first is creating risk management models for lending and credit risk management; the second is in fraud prevention, where AI systems identify, track and flag potential threats.

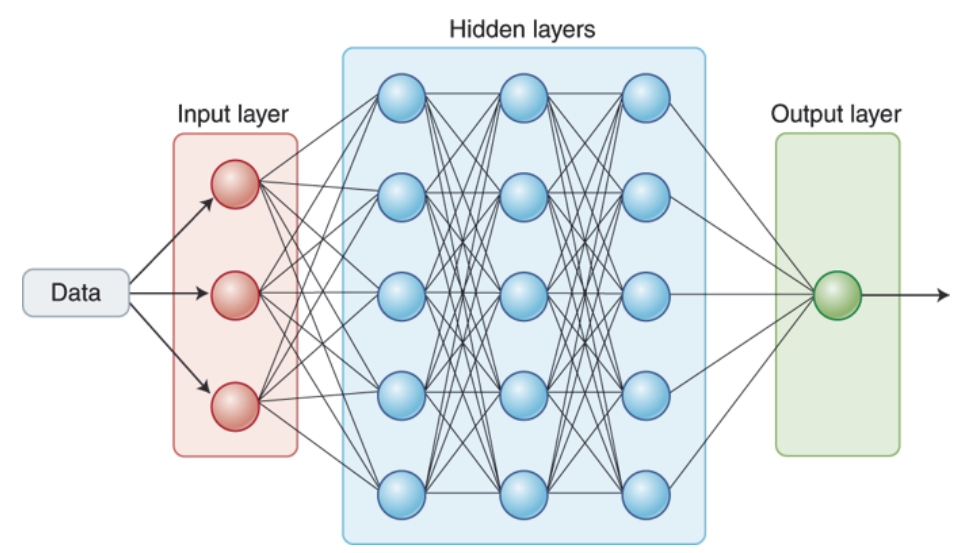

Figure 5. Deep Learning

Source: Google Images (2019)

\subsubsection{Neuron}

A neuron is just a MATHEMATICAL FUNCTION that takes inputs (the outputs of the neurons pointing to it) and returns outputs. These outputs serve as inputs for the next layer, and so on until we get to the final, output layer, which is the actual value we return. For each set of inputs, the Neural Network's goal is to make each of its outputs as close as possible to the actual expected values. A neuron will take an input vector, and basically do three things to it:

- Multiply it by a weights vector.

- Add a bias value to that product. 
- Apply an activation function to that value.

We get a new vector as each layer's output; feed it to the next layer as inputs, and so on. Bias is a constant which helps the model in a way that it can fit best for the given data. Each layer in a Neural Network has an activation function.
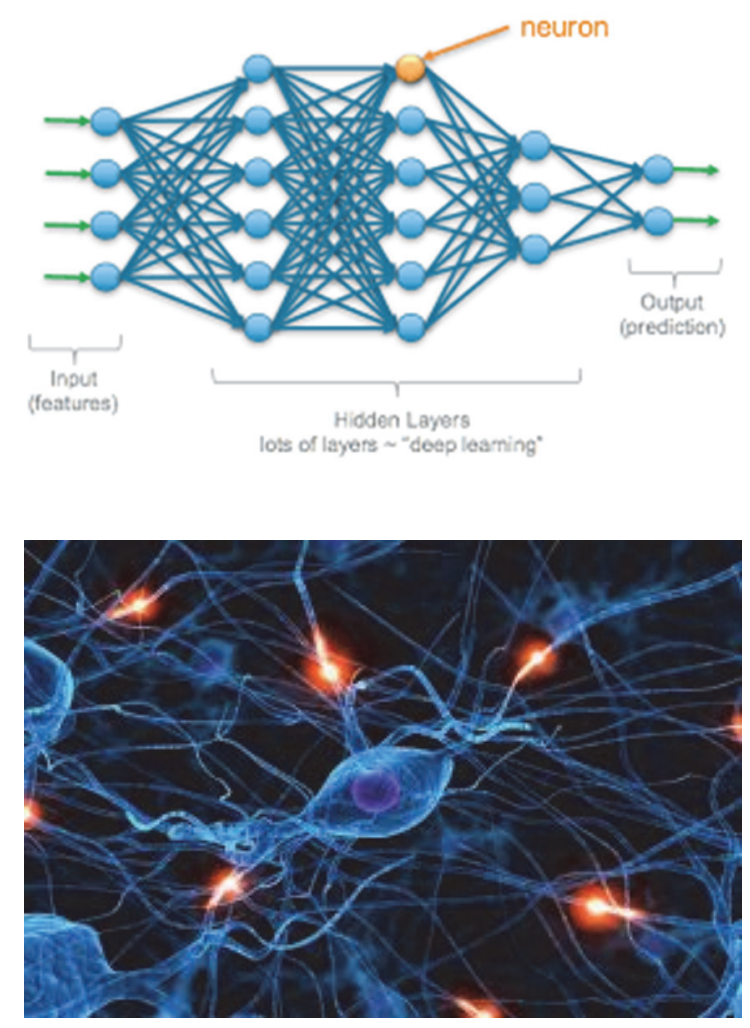

Figure 6. Neuron

Source: Google Images (2019)

\subsubsection{Neural Network}

A Neural Network is a Machine Learning model that, given certain input and output vectors, will try to "fit" the outputs to the inputs. What this means is, given a set of observed instances with certain values we wish to predict, and some data we have on each instance, it will try to generalize those data so that it can predict the values correctly for new instances of the problem. And we finally got to the core of our business: that's what activation functions do. We'll typically use non-linear functions as activation functions. This is because the linear part is already handled by the previously applied product and addition. 


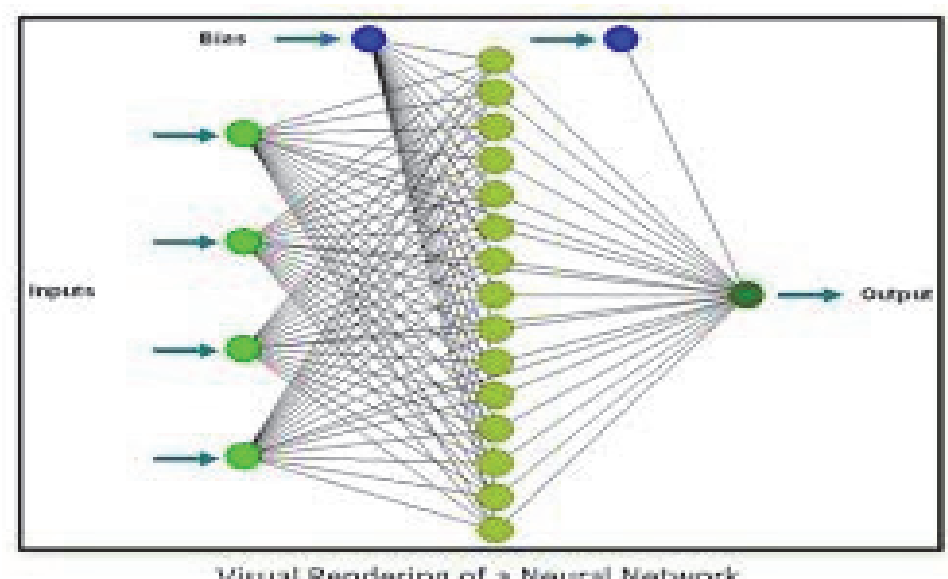

Visual Rendering of a Neural Network

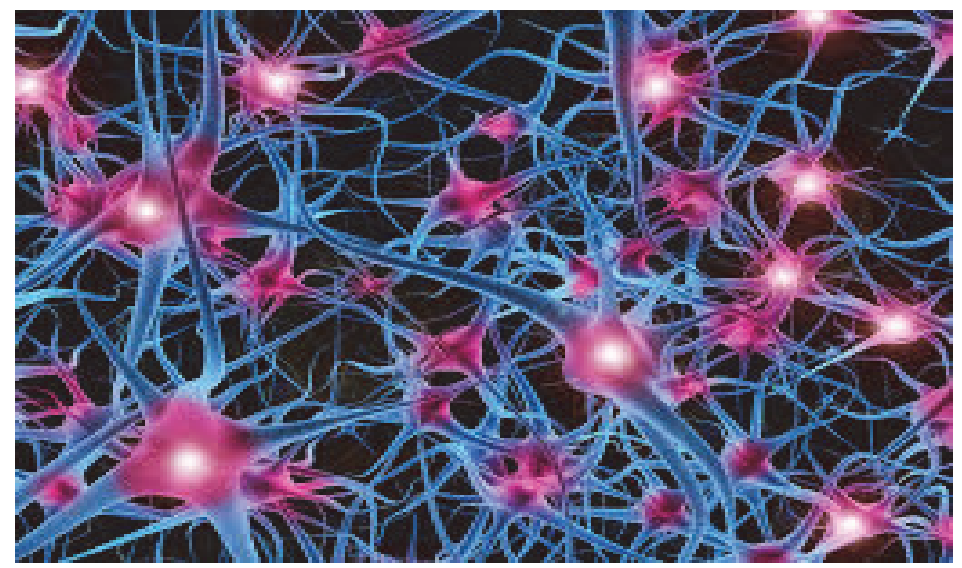

Figure 7. Neural Network

Source: Google Images (2019)

\subsection{Methods}

Model complexity has increased; models are being used in many areas of banking and finance applications. This scrutiny was kick-started by the aftermath of the global financial crisis, in which it became apparent that at least some incumbent models at the time were unfit for purpose, which was exacerbated by a lack of appropriate governance around models and their use.

The supervisory reaction has drastically increased regulatory scrutiny for how models are built, approved and maintained. In 2011, the Federal Reserve and the Office of the Comptroller of the Currency published the supervisory guidance on Model Risk Management which mandated banks to submit documentation on model risk management policies and practices. This is a regulatory standard for model risk management and the regulators around the globe picked up the challenge. In 2017, the European Central Bank (ECB) published guidelines which expect financial institutions to have a well-developed model risk management framework in place, and to apply it to all models used for business decision-making; especially those pertaining to capital requirements. In the United Kingdom (UK), the Prudential Regulation Authority (PRA) is also heavily focused on model risk; with stress testing models getting a lot of attention. In 2018, the PRA published a supervisory statement on model risk management for stress testing (SS3/18), covering firms' development, implementation and use of stress testing models. Algorithmic trading has also seen increased regulatory focus in the UK. The PRA published a supervisory statement on Algorithmic Trading (SS5/18) outlining expectations on the risk management and governance of Algorithmic trading, where machine learning models are increasingly being used to facilitate automated trading decisions. These developments are mirrored in many other jurisdictions around the world. 
Most banks today are quite familiar with machine learning algorithms that learn by example from historical data and are used to predict outcomes and uncover patterns that are not easily spotted by humans. However, not all classes of machine learning algorithms are recent developments; many have been used in the banking and financial services industry for decades. These include basic statistical methods and techniques like linear and logistic regression and rule-based modeling. These traditional methods are relatively simple, well-understood by practitioners and easily explainable and interpretable. However, recently, the U.S. Congress has intensively criticized AI and ML as the Technological or Scientific advancement that lack transparency subject to Data Manipulations with unregulated outcomes that does not gives Companies possibilities to make well informed, $\mathrm{AI}$ and ML Algorithms are running in secrecy, the Black Box and beyond the control of the User and the Builder, Technology without having Human Independent supervisor, oversight or regulation, that the Technology cannot trusted anymore, AI \& ML algorithms gives bad recommendations, care should be taken about Final Ranking of the content among others. To increase TRANSPARENCY and STABILITY in the Activation Functions output (final ranking of the content) so as to help policy makers, Companies and decision makers with optimum the right choice among the final activation functions to enable them make well informed decision, the paper propose the following Advanced Stochastic Algorithms. Also, this propose algorithm will logically make an attempt to answer the questions: "why is that one works better than the other?", "how do we know which one to use?" "is it hardcore maths?" and so on.

\subsubsection{The Basic Idea}

The basic IDEA was due to U.S. Congress meeting entitled, "Optimizing for Engagement: Understanding the Use of Persuasive Technology on Internet Platforms" held on Tuesday, June 25, 2019 by the Subcommittee on Communications, Technology, Innovation, and the Internet chaired by Sen. John Thune, R-S.D, with aim to find appropriate policy on algorithms' transparency or explanation as regards to its decision-making and machine learning on internet platforms might be influencing the public. The Author attempted to INCORPORATE TRANSPARENCY and STABILITY in the existing SET of Activation Functions thereby opening the Deep Learning Artificial Neural Network's "BLACK-BOX" in the Areas of Banking and Finance.

The Author observed that the NON-LINEAR Activation Functions were introduced into Deep Learning Artificial Neural Networks to introduce NON-LINEARITY into the system. Thus, this implies Activation Functions are the BRAIN behind the successful functioning of any Deep Learning Artificial Neural Network.

To INCORPORATE STABILITY in the existing the Deep Learning Artificial Neural Network System REFERENCE to out AI-DATA SET, the Author considered the set of existing Activation Functions thereby picking SIGMOID. Sigmoid is a fat-tailed Probability Distribution, however, the Author thinks on how to incorporate LOW-PROBABILITY, HIGH-IMPACT effects (additional fat-tailed effects) in it.

Jamilu (2015) proposed Jameel's Contractional-Expansional Stress Method that depends on JAMEEL'S CRITERION to incorporate fat-tail effects in BANKING and ECONOMIC Stochastic Models, particularly the Logistic Regression (LOGIT) and PROBIT Models as shown in the figure below: 


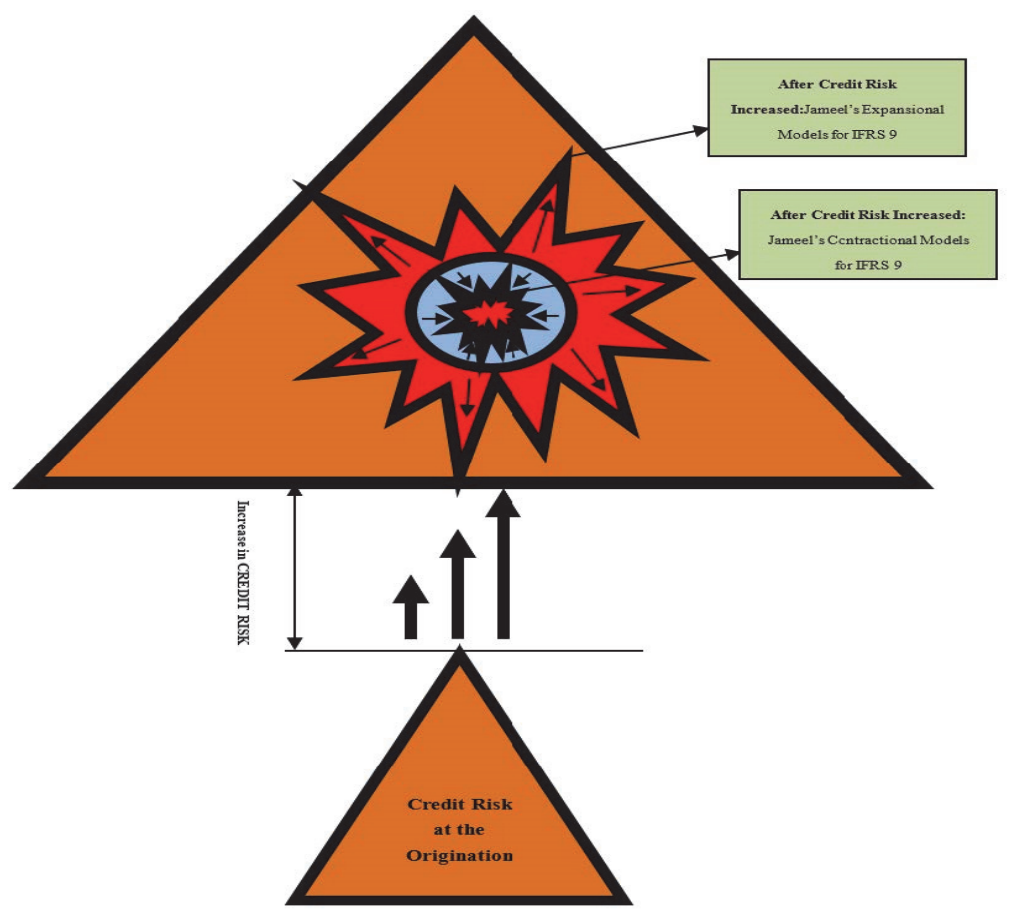

Figure 8. The Basic Idea

Source: The Author (2018)

The Author applied the above Idea to Sigmoid Activation Function:

Sigmoid/Logistic Function (famous function in the Neural Network):

$$
y=\frac{1}{1+e^{-x}} ;-\infty<x<+\infty
$$

Thus, we have the following Stressed Sigmoid Types:

Stressed Sigmoid Type A:

$$
\frac{1}{1+\exp \mu_{A}(-x) \pm \sigma_{A} f(x)}
$$

Stressed Sigmoid Type B:

$$
\frac{1}{1+\exp \mu_{A}(-x) \pm f(x)}
$$

Stressed Sigmoid Type C:

$$
\frac{1}{1+\exp (-x) \pm \sigma_{A} f(x)}
$$

Stressed Sigmoid Type D:

$$
\frac{1}{1+\exp (-x) \pm f(x)}
$$




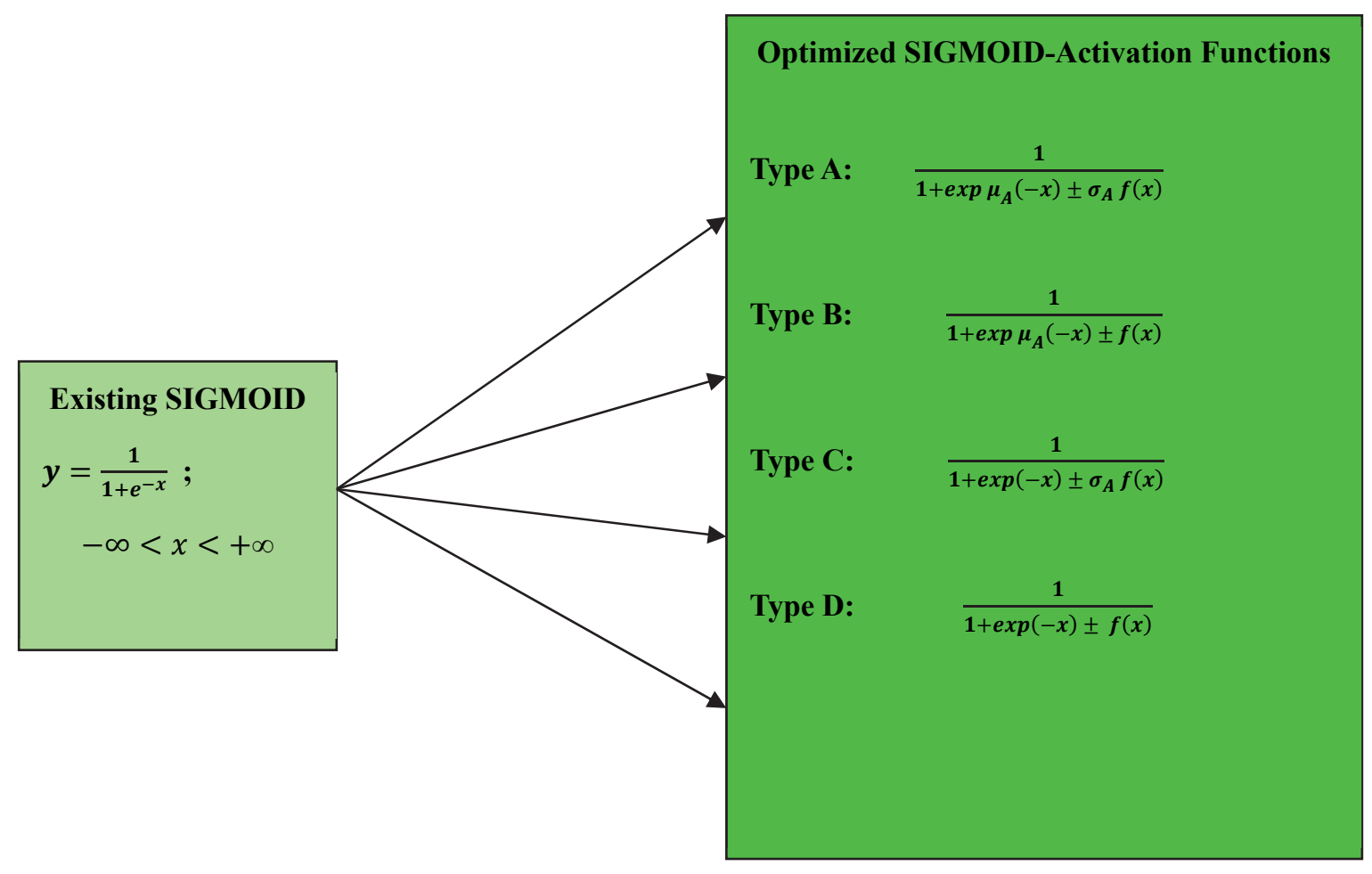

Figure 9. Stressed Sigmoid-Activation Functions

Source: The Author (2019)

Where, the Geometric Mean $\left(\mu_{A}\right)$ and Volatility $\left(\sigma_{A}\right)$ of the Macroeconomic Indicators and Task. $f(x)$, the Fat-tailed Stochastic Function satisfied Jameel's Criterion.

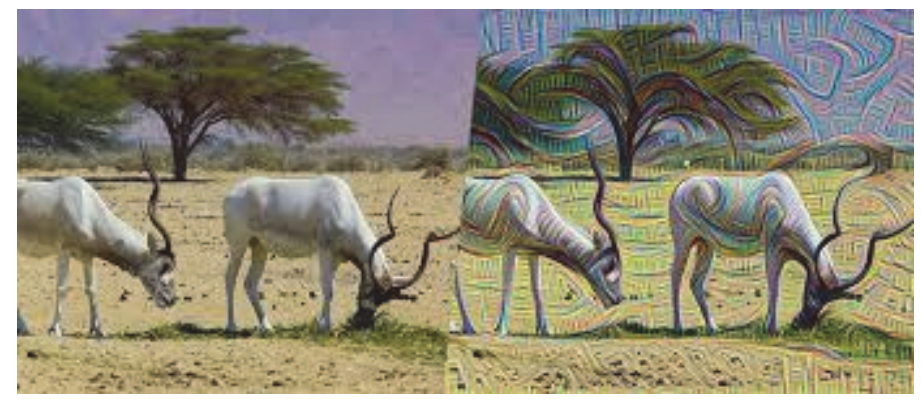

Figure 10. Expected Stressed Sigmoid Output

Source: Google Images (2019)

For instance, the Ultimate Goal is to drastically minimize the NOISES in the Right Photo of figure 10 above.

After the Sigmoid was Stressed, the Author continued to brainstorm with available Literature concluded that the existing set of Activation Functions were introduced into the Deep Learning Artificial Neural Network System through the "WINDOW" not properly through the "LEGITIMATE DOOR". 


\subsubsection{Existing Activation Functions and their Relationships with AI-ML-Purified Data Set}

The existing Activation Functions has no correlation(s) in whatsoever with the AI-ML-Purified Data Set under consideration, in fact, their choices has no definite Rule of Thumb; it is just Trial and error as shown in the figure below:
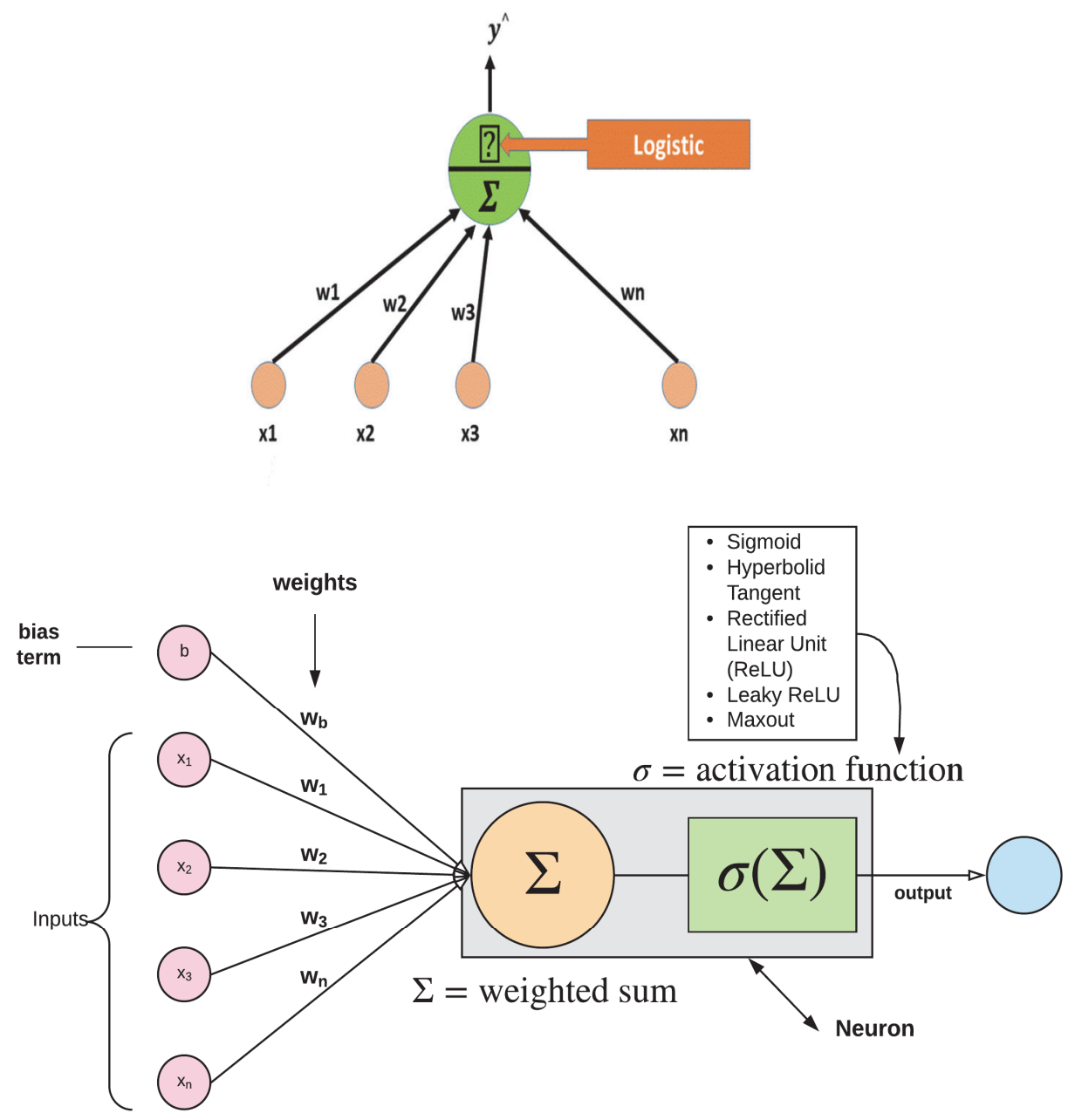

Figure 11. Activation Functions in ANN

Source: Google Images (2019) 
3.2.3 Who are the Competent and Eligible Activation Functions for a successful Neural Network

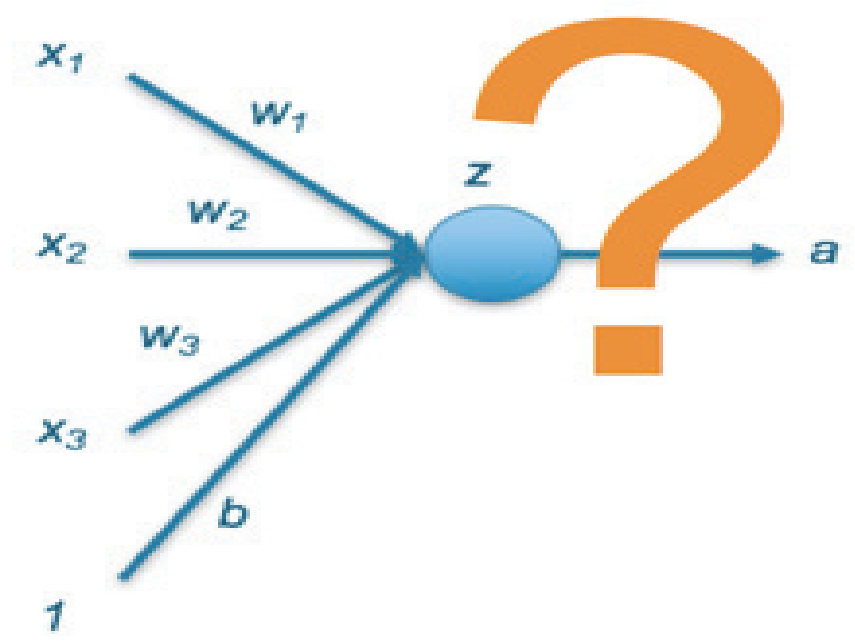

Figure 12. Question of Competent and Eligible Activation Functions

Source: Google Images (2019)

ACTIVATION FUNCTIONS Allows Artificial Neural Network to learn complex functional mappings from

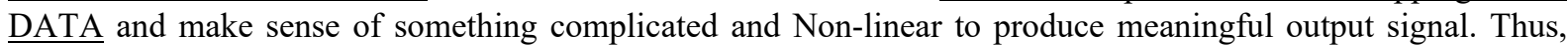
should not be a Trial and error or a Black-Box Assumptions.

Biologically, Neuron performs three basic functions, namely: Receive signals (or information); Integrate incoming signals (to determine whether or not the information should be passed along); and, Communicate signals to target cells (other neurons or muscles or glands). However, this cannot be done successfully without action of a Nonlinear Function (Brain of Neuron) residing in a Neuron of Human Brain EMANATED from incoming signals (information). This Non-linear Function (Brain of Neuron) residing in a Neuron of Human Brain EMANATED from incoming signals is what we called Activation Function of a Deep Learning Artificial Neural Network.

The Author PROPOSED that the Competent and Eligible Activation Functions for the successful conduct of Artificial Neural Networks are the Activation Functions EMANATED from the AI-ML-Purified Data Set under consideration satisfied AI-ML-Jameel's Stochastic or Deterministic Criterion because of the following SCIENTIFIC FACTS:

(1) They EMANATED from the referenced AL-ML-Purified Date Set and satisfied AI-ML-Jameel's Stochastic and or Deterministic Criterion;

(2) They have a very strong (if not perfect) CORRELATION with the referenced AI-ML-Purified Data Set. A link between the Data Set and Activation Functions MUST be strongly established since Artificial Neural network uses past historical data to predict the future of a given task with the aid of machines;

(3) They relate better to the referenced AI-ML-Purified Data Set then the existing Assumed and Trial and error Activation Functions;

(4) They indeed describe the distribution of our referenced AI-ML-Purified Data Set, which is a listing or function showing all the possible values (or intervals) of the data and how often they occur;

(5) They represent real, virtual and un-virtual information about our referenced AI-ML-Purified Data Set;

(6) They indeed captured the Symmetric, Left Skewed, Right Skewed, Mesokurtic, Leptokurtic, and Platykurtic properties of our referenced AI-ML-Purified Data Set;

(7) They contain real, virtual and un-virtual information related to Measures of variability (the range, interquartile range, and standard deviation) and Measures of Central Tendency (Mean, Mode and Median, Minimum and Maximum) of our referenced AI-ML-Purified Data Set; 
(8) They capture real, virtual and un-virtual information about the Correlation (autocorrelation) among the elements in our referenced AI-ML-Purified Data Set;

(9) In the case of Bivariate AI-ML-Data Set, they captured real, virtual and un-virtual information about Measures of Association (Covariance and Correlation) of our referenced AI-ML-Purified Data Set;

(10) They capture real, virtual and un-virtual information whether or not the parameters of our referenced AI-MLPurified Data Set are constant over time; and,

(11) They also captured the presence of outliers in our referenced AI-ML-Purified Data Set under consideration.

Referenced AI-ML-Purified Data Set means our referenced Artificial Neural Network Data Set shall possess the following QUALITIES:

a. Accuracy and Precision

b. Legitimacy and Validity

c. Reliability and Consistency

d. Timeliness and Relevance

e. Completeness and Comprehensiveness

f. Availability and Accessibility

g. Granularity and Uniqueness

Therefore, the author proposed that the practice of "TRIAL AND ERROR" choice of Assumed Activation Functions should be abandoned, however, the choice of Activation Functions should follow a "DEFINITE RULES", and thus, the Author enhanced "JAMEEL'S CRITERION (2015)" to proposed "JAMEEL'S ANNAF STOCHASTIC CRITERION (2019)" as follows:

3.2.4 Proposed Jameel's ANNAF Stochastic Criterion

ANNAF means Artificial Neural Network Activation Functions.

Under this criterion, we run the goodness of fits test on our referenced PURIFIED AL-ML-DATA SET such that:

(i) We accept if the Average of the ranks of Kolmogorov Smirnor, Anderson Darling and Chi-squared is less than or equal to Three (3);

(ii) We must choose the fat-tailed Probability Distribution $f(x)$ follows by our referenced PURIFIED ALML-DATA SET ITSELF regardless of its Rankings;

(iii) If there is tie, we include both the fat-tailed Probability Distributions in the selection;

(iv) At least Two (2) fat-tailed Probability Distributions must be included in the selection;

(v) We select the most occur Probability Distribution as the qualify candidate in each case of test of goodness of fit on our referenced PURIFIED AL-ML-DATA SET;

(vi) Backward Propagation Axiom: Having chosen the most qualified fat-tailed Probability Distribution in the final round up selection for instance, $f_{\text {final }}(x)$, then $f_{\text {final }}(x)$ SHALL BE MONOTONE

CONTINUOUSLY DIFFERENTIABLE FUNCTION. Particularly if $f_{\text {final }}(x)$ is NOT

DIFFERENTIABLE then DISCARD it and repeat (i) to (vi) until we have a MONOTONE DIFFERENTIABLE FUNCTION. Note the Approach is from TOP-BOTTOM process;

(vii) Criterion Enhancement Axiom:Thode (2012) intensively discussed about the Best Goodness of Fit Tests such as Kolmogorov Smirnov (KS) Test, Anderson-Darling Test, Jarque and Bera (JB) Test, Shapiro Wilk (SW) Test, Cramer-Von Mises Test, Pearson ( $\chi^{2}$ Godness of Fit) Test, Lilliefors Corrected KS Test, D’AgostinoSkewness Test, Anscombe-Glynn Kurtosis Test, D’Agostino-Pearson and Omnibus

Test. Let $\left\{T_{1}, T_{2}, \ldots, T_{n}\right\}$ be the set of such Best Goodness of Fit Tests, $\left\{x_{1}, x_{2}, \ldots, x_{n}\right\}$ be their RANKS 
respectively then the generality of (i) can be re-expressed (or enhanced) if $\frac{\left(x_{1}+x_{2}+\ldots+x_{n}\right)}{n} \leq a$, where $0<a \leq n, n \in N$ or equivalently, $x_{1}+x_{2}+\ldots+x_{n} \leq a n ;$ and,

(viii)Unit Axiom: let $f_{\text {final }}(x)$ be such that it satisfied axioms (i) to (iv) and or (vii). Let $\left\{r_{1}, r_{2}, \ldots, r_{n}\right\}$ be the ranks of fitness test of $f_{\text {final }}(x)$ obtained from the tests $\left\{T_{1}, T_{2}, \ldots, T_{n}\right\}$ respectively then if $\forall i \in\{1,2, \ldots, n\}, \quad r_{i}=1$ regardless of other factors. Consequently, if for all fitness test runs, turn out to be the same $f_{\text {final }}(x)$ then $f_{\text {final }}(x)$ will gives Deep Learning Artificial Neural Networks $\underline{\text { SUPER- }}$

\section{INTELLIGENT CAPABILITIES.}

3.2.5 Proposed Jameel's Stochastic Lemma

All the TOP-RANKED Fat-tailed Monotone Continuously Differentiable Stochastic Functions EMANATED from referenced AI-ML-Purified Data satisfied Proposed Jameel's Stochastic ANNAF Criterion are EXCELLENT STOCHASTIC ACTIVATION FUNCTIONS to perform well-informed Forward and Backward Propagations of an Artificial Neural Network.

\subsubsection{Stocks Advanced Optimized Activation Functions}

The Ranking of the Advanced Optimized Activation Functions was obtained using Jameel's Criterion (second version). The author considered Eleven (11) out of Fifty (50) World's Biggest Public Companies by FORBES as of 2015 Ranking Regardless of the platform in which they are listed, Time Series (Short or Long Terms), Old or Recently Listed Companies. The Ranking can be seen as follows:

Log - Logistic (3P) Probability Distribution (1 $\left.{ }^{\text {st }}\right)$ :

$$
f(x ; \mu, \sigma, \xi)=\frac{\left(1+\frac{\xi(x-\mu)}{\sigma}\right)^{-(1 / \xi+1)}}{\left[1+\left(1+\frac{\xi(x-\mu)^{-1 / \xi}}{\sigma}\right)\right]^{2}} ; x \geq \mu
$$

Cauchy Probability Distribution $\left(2^{\text {nd }}\right)$ :

$$
f(x ; \mu, \sigma, \pi)=\left(\pi \sigma\left(1+\left(\frac{x-\mu}{\sigma}\right)^{2}\right)\right)^{-1} ;-\infty<x<+\infty
$$

Pearson 5 (3P) Probability Distribution $\left(3^{\text {rd }}\right)$ :

$$
f(x ; \alpha, \beta, \gamma)=\frac{\exp \left(-\frac{\beta}{(x-\gamma)}\right)}{\beta \Gamma(\alpha)\left(\frac{(x-\gamma)}{\beta}\right)^{\alpha+1}} ; \alpha, \beta>0
$$

Burr (4P) Probability Distribution (4th): 


$$
f(x ; \alpha, \beta, \gamma)=\frac{a k\left(\frac{x-\gamma}{\beta}\right)^{\alpha-1}}{\beta\left(1+\left(\frac{x-\gamma}{\beta}\right)^{\alpha}\right)^{k+1}} ; \alpha, \beta, k>0
$$

Fatique Life (3P) Probability Distribution $\left(5^{\text {th }}\right)$ :

$$
f(x ; \alpha, \beta, \gamma)=\frac{\sqrt{\frac{(x-\gamma)}{\beta}}+\sqrt{\frac{\beta}{(x-\gamma)}}}{2 \alpha(x-\gamma)} \Phi\left(\frac{1}{\alpha}\left(\frac{\sqrt{x-\gamma}}{\beta}-\sqrt{\frac{\beta}{x-\gamma}}\right)\right) ; \alpha, \beta, \gamma>0
$$

Inv.Gaussian (3P) Probability Distribution ( $\left.6^{\text {th }}\right)$ :

$$
f(x ; \mu, \lambda, \gamma)=\sqrt{\frac{\lambda}{2 \pi(x-\gamma)^{3}}} \exp \left(-\frac{\lambda(x-\gamma-\mu)^{2}}{2 \mu^{2}(x-\gamma)}\right) ; \lambda, \mu, \gamma>0
$$

Dagum (4P) Probability Distribution $\left(7^{\text {th }}\right)$ :

$$
f(x ; \gamma, \beta, \alpha)=\frac{a k\left(\frac{x-\gamma}{\beta}\right)^{a k-1}}{\beta\left(1+\left(\frac{x-\gamma}{\beta}\right)^{\alpha}\right)^{k+1}} ; k, \alpha, \beta>0
$$

Lognormal (3P) Probability Distribution $\left(8^{\text {th }}\right)$ :

$$
f(x ; \mu, \sigma, \gamma)=\frac{\exp \left(-\frac{1}{2}\left(\frac{\ln (x-\gamma)-\mu}{\sigma}\right)^{2}\right)}{(x-\gamma) \sigma \sqrt{2 \pi}} ; \gamma<x<+\infty
$$

Note that the paper did not check the Monotone Differentiability of the functions presented above. However, the market trends are very volatile, many things had happened from $2019-2015$. The distributions time series Data was from 2014 - 1990, Jamilu (2015) showed this. Different results may be obtained when conducted in the year 2019. Anyway, the paper adopted the fat-tailed probability distributions obtained as per as 2015 .

\section{Conclusion}

The U.S. Congress, professionals, and the general public strongly believed that AI and ML have shortcomings despite the overwhelming advancement in Banking and Finance Industries, electronic trading platforms, medical diagnosis, robot control, entertainment, education, health, and commerce.

To address the FEARS raised by the U.S. Congress, the paper attempted to increase TRANSPARENCY and STABILITY in terms of Activation Functions output to help the policymakers, companies, and decision-makers with right Activation Functions using Definite Rules to enable them to make a well-informed decision.

To open the "Black Box" of Deep Learning Artificial Neural Network Systems, we need MONOTONE DIFFERENTIABLE FAT-TAILED PROBABILITY DISTRIBUTIONS EMANATED from our REFERENCED AI-ML-Purified Data Set satisfied Jameel's Stochastic ANNAF Criterion.

This will strongly establish a very high (almost perfect) correlation between the AI-ML-Purified Data Set and the set of Stochastic Activation Functions of Deep Learning Artificial Neural Network Systems. The bottom line is that “the Deep Learning Artificial Neural Network Stochastic Activation Functions Satisfied Jameel's Criterion 
would depend on REFERENCED AI-ML-Purified Data Set" to successfully perform well-informed Deep Learning Artificial Neural Network Learning processes.

Furthermore, this research REVEALED that the Advanced Activation Functions satisfied Jameel's ANNAF Stochastic or Deterministic Criterion depends on the REFERENCED PURIFIED AI DATA SET, TIME CHANGE and AREA OF APPLICATION (acronym DTA) as shown in the figure below:

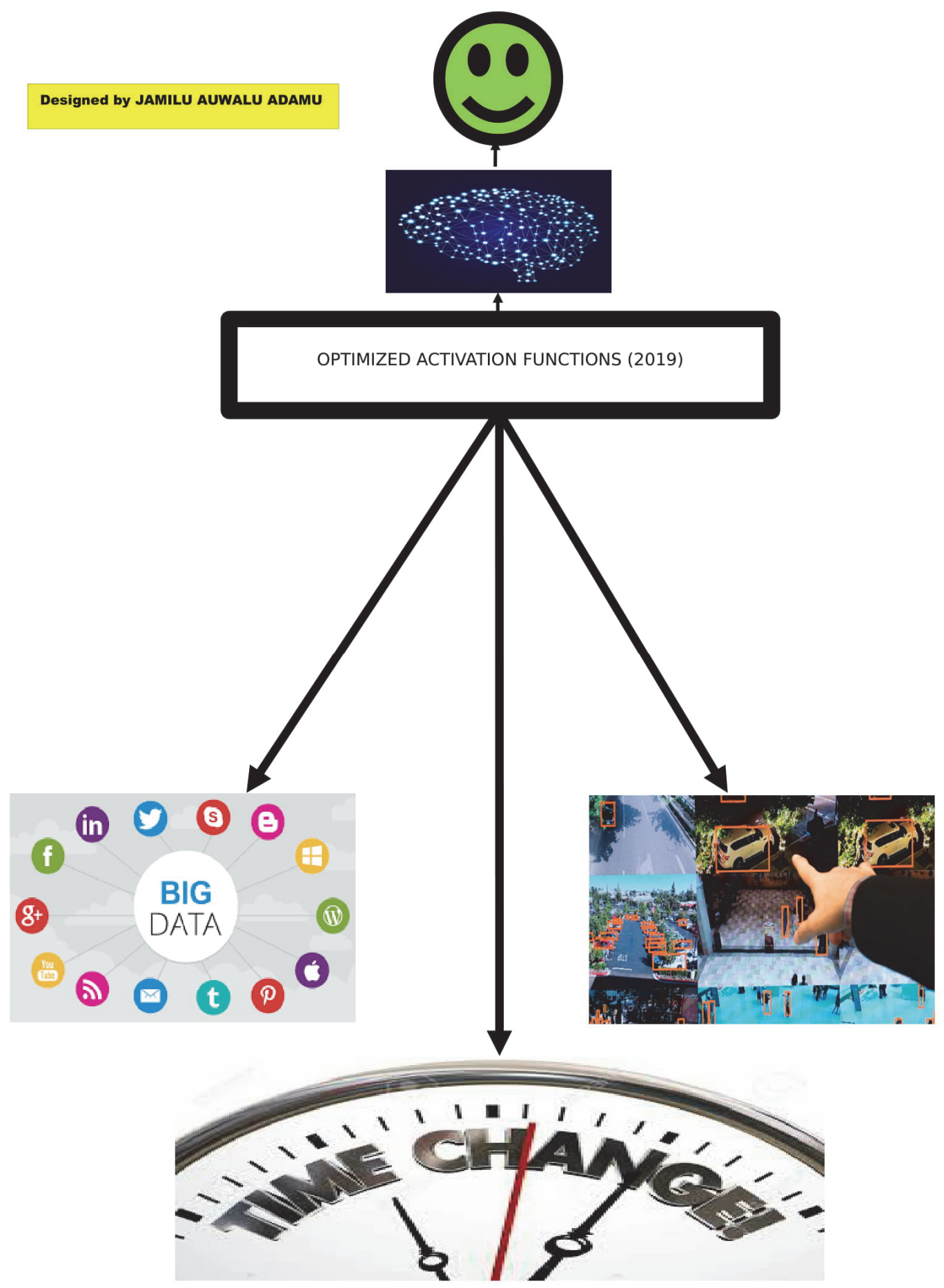

Figure 13. Optimized Activation Functions depends on AI DATA, TIME CHANGE \& AREA OF APPLICATION

Source: The Author (2019) 
This is against the traditional Trial and Error set of assumed Activation Functions, INDEPENDENT of DTA.

Research for better activation functions is still ongoing, however, the FUTURE of AI and ML lies on the emphasis given to the DEFINITE RULES of choosing Right Activation Functions considering the impact of LOWPROBABILITY, HIGH-IMPACT EFFECTS, basically, attention given to FAT-TAILED EFFECTS.

This paper was SUMMARIZED in the following FIVE (5) Youtube Videos:

(1) https://www.youtube.com/watch?v=nth3cJqgFts\&t=5s

(2) https://www.youtube.com/watch?v=lcyR4TCOBFw

(3) https://www.youtube.com/watch?v=15NgJh71KRQ\&t=3s

(4) https://www.youtube.com/watch?v=6emMNluHMZg

(5) https://www.youtube.com/watch?v=IlDTNWc7C-8

Declaration of Interest: The Author reports no conflict of Interest. The views expressed in this paper are those of the Author and not his current employer.

\section{Acknowledgments}

This research paper was a sequel to my Ph.D research work research findings extension with Ahmadu Bello University, Zaria, Nigeria. Firstly, I would like to thank the Federal Government of Nigeria through the National Mathematical Centre for releasing me for my Ph.D study. Second, I appreciate the efforts of Lan Smith, Assistant Editor, Risk, and Financial Management Journal, USA.

Also, I appreciate the Anonymous Reviewer(s) for his/her constructive criticism to improve the quality of this manuscript, however, if fact, $\mathrm{He} /$ she suggested to include Appendix A, B, and C for wider readability of the manuscript especially readers without strong Mathematical background.

Finally, I thank my mum (Hajiya Hauwa Ahmad), my lovely wife Halima and children Islam and Salman, they are the sources of my Creativity, Energy, and Aspiration.

\section{References}

Aman, D., \& Payal, P. (2019). Analysis of Non-Linear Activation Functions for Classification Tasks Using Convolutional Neural Networks. Recent Patents on Computer Science Journal, 12(3). https://doi.org/ $10.2174 / 2213275911666181025143029$

Aman, D., \& Payal, P. (2019). Analysis of Non-Linear Activation Functions for Classification Tasks Using Convolutional Neural Networks. Recent Patents on Computer Science, 12(3). https://doi.org/10.2174/2213275911666181025143029

Artist Hans Hoffman wrote, "The ability to simplify means to eliminate the unnecessary so that the necessary may speak." Retrieved fromhttps://www.brainyquote.com/quotes/hans_hofmann_107805

Barnaby, B. et. at (2016). Complying with IFRS 9 Impairment Calculations for Retail Portfolios, Moody's Analytics Risk Perspectives, the convergence of Risk. Finance, and Accounting, VII.

Bellotti, T., \& Crook, J. (2012). Loss Given Default Models Incorporating Macroeconomic Variables for Credit Cards. International Journal of Forecasting, 28(1), 171-182. https://doi.org/10.1016/j.ijforecast.2010.08.005

Ben, S. (2019). Model Risk Management for Deep Learning and Alpha Strategies, BNP Paribas Asset Management, Quant Summit 2019.

Burton, G. M. (2009). The Clustering of Extreme Movements: Stock prices and the Weather, Princeton University, AtanuSaha, Alixpartners, Alex Grecu, Huron Consulting Group, CEPS working paper No. 186 February, 2009.

Casper, H. (2019). says "Better optimized neural network; choose the right activation function, and your neural network can perform vastly better". Retrieved from https://mlfromscratch.com/neural-networksexplainerd/\#/

Chigozie, E. N. et al. (2018). Activation Functions: Comparison of Trends in Practice and Research for Deep Learning. Retrieved fromhttps://arxiv.org/pdf/1811.03378.pdf

Chigozie, E. N. et al. (2018). Activation Functions: Comparison of Trends in Practice and Research for Deep Learning, Preprint.

Daniel, P. (2006). Estimating Probabilities of Default for German Savings Banks and Credit Cooperatives, University of Applied Sciences, Mainz, Ander Bruchspitze 50, D - 55122 Mainz 
David, M. R. (2012). Simulating Default Probabilities in Stress Scenarios, Presented to the PRMIA Global Risk Conference, New York, NY, May 14, 2012.

David, R. (2019). Responding to the AI Challenge Learning from Physical Industries, @2019 The Mathworks, Inc.

Djork-Arne Clevert, Thomas Unterthiner \& Sepp Hochreiter (2016). FAST AND ACCURATE DEEP NETWORK LEARNING BY EXPONENTIAL LINEAR UNITS (ELUS), Published as a conference paper at ICLR 2016

Jamilu, A. A. (2015). Banking and Economic Advanced Stressed Probability of Default Models. Asian Journal of Management Sciences, 3(08), 10-18.

Jamilu, A. A. (2015). Estimation of Probability of Default using Advanced Stressed Probability of Default Models, Ongoing Ph.D Thesis, Ahmadu Bello University (ABU), Zaria, Nigeria.

Jamilu, A. A. (2016). Reliable and Sophisticated Advanced Stressed Crises Compound Options Pricing Models. Management and Organizational Studies, 3(1), 39-55. https://doi.org/https://doi.org/10.5430/mos.v3n1p39.

Jamilu, A. A. (2017). An Introduction of Jameel's Advanced Stressed Economic and Financial Crises Models and to Dramatically Increasing Markets Confidence and Drastically Decreasing Markets Risks. International Journal of Social Science Studies, 4(3), 39-71. https://doi.org/https://doi.org/10.11114/ijsss.v4i3.1326

Jamilu, A. A. (2017). Jameel's Criterion and Jameel's Advanced Stressed Models: An Ideas that Lead to NonNormal Stocks Brownian Motion Models. Noble International Journal of Business and Management Research, 1(10), 136-154. URL: http://napublisher.org/?ic=journals\&id=2.

Jamilu, A. A. (2017). Jameel's Criterion and Jameel's Advanced Stressed Models: An Ideas that Lead to NonNormal Stocks Brownian Motion Models. Noble International Journal of Business and Management Research, 1(10), 136-154. URL: URL: http://napublisher.org/?ic=journals\&id=2

Jamilu, A. A. (2018). Jameel's Dimensional Stressed Default Probability Models are Indeed IFRS 9 Complaint Models. Journal of Economics and Management Sciences, 1(2), 104-114. https://doi.org/10.30560/jems.v1n2p102.

Joonho, L. et al. (2019), ProbAct: A Probabilistic Activation Function for Deep Neural Networks, Preprint. Under review.

Klambauer, et, al. (2017). Self-Normalizing Neural Networks, Institute of Bioinformatics, Johannes Kepler University Linz, Austria.

Lichman. (2013). UCI machine learning repository. Retrieved from http://archive. ics. uci. edu/ml 901

M, \& Van, N. (1968). Fractional Brownian Motions, Fractional Noises and Applications (M \& Van Ness (1968)). SIAM Review, 10, 422-437

Mohit, G. et al. (2019). Learning Activation Functions: A new paradigm for understanding Neural Networks. Proceedings of Machine Learning Research, 101, 1-18.

Nair, et al. (2010). Rectified linear units improve restricted boltzmann machines, ICML'10 Proceedings of the 27th International Conference on International Conference on Machine Learning Pages 807-814, Haifa, Israel June 21 - 24, 2010.

Nassim, N. T. (2007). Black Swans and the Domains of Statistics. American Statistician, 61(3).

Nassim, N. T. (2009). Errors, Robustness, and Fourth Quadrant, New York University Polytechnic Institute and Universa Investment, United States. International Journal of Forecasting, 25, 744-759.

Nassim, N. T. (2010). Convexity, Robustness, and Model Error inside the "Black Swan Domain", Draft Version, September, 2010.

Nassim, N. T. (2010). Why Did the Crisis of 2008 Happen, Draft (3rd ed.), August, 2010.

Nassim, N. T. (2011). A Map and Simple Heuristic to Detect Fragility, Antifragility, and Model Error (1st ed.).

Nassim, N. T. (2011). The Future has Thicker Tails Past: Model Error as Branching Counterfactuals, presented in Honor of Benoit Mandelbrot's at his Scientific Memorial, Yale University, April, 2011.

Nassim, N. T. (2012). The Illusion of Thin - Tails under Aggregation, NYU - Poly, January, 2012

Nassim, N. T. et al. (2009). Risk Externalities and Too bid to Fail, New York University Polytechnic Institute, 11201, New York, United States.

Onali, E., \& Ginesti, G. (2014). Pre-adoption Market Reaction to IFRS 9: A Cross-country Event-study. Journal 
of Accounting and Public Policy, 33(6), 628-637.

Peter Martey Addo et al. (2018). Credit Risk Analysis using Machine and Deep Learning Models. Risks Journal, Risks, 6(38). https://doi.org/10.3390/risks6020038

Ram, Ananth, et al. (2019). Opening the "Black Box". The Path to Deployment of AI Models in Banking, White Paper, DataRobot and REPLY AVANTAGE.

Reney, D. E., \& Michael, R. M. (2016). Forecasting of Stock Prices using Brownian Motion - Monte Carlo Simulation. Proceedings of the 2016 International Conference on Industrial Engineering and Operations Management. Kuala Lumpur, Malaysia, March 8-10, 2016.

Schoenholz, et al. (2017). DEEP NEURAL NETWORKS AS GAUSSIAN PROCESSES, Published as a $\begin{array}{llllll}\text { conference paper } & \text { at } & \text { ICLR } & \text { Retrieved }\end{array}$ file://C:/Users/pc/Downloads/Deep Neural Networks as Gaussian Processes.pdf

Sebastian, R. (2018), STAT 479: Machine Learning Lecture Notes. Retrieved from http://stat.wisc.edu/ sraschka/teaching/stat479fs2018/.

Sebastian, U. (2017). Neural Network Architectures and Activation Functions: A Guassian Process Approach, Technical University Munich, 2017.

Soufiane, H. et al. (2019). On the Impact of the Activation Function on Deep Neural Networks Training. Retrieved from https://arxiv.org/pdf/1902.06853.pdf

Soufiane, H. et al. (2019). On the Impact of the Activation Function on Deep Neural Networks Training, Proceedings of the 36 th International Conference on Machine Learning, Long Beach, California, PMLR 97, 2019.

Spreedhar, T. B. et al. (2004). Forecasting Default with the KMV - Merton Model, University of Michigan, Ann Arbor MI 48109.

Steven, R. D. (). Stochastic Processes and Advanced Mathematical Finance, The Definition of Brownian Motion and the Wiener process, Department of Mathematics, 203 Avery Hall, University of Nebraska-Lincoln, Lincoln, NE 68588-0130.

Sven-Patrik, H. (). Machine Learning, Deep Learning, Experimental Particle Physics, University of Glasgow.

Tidaruk, A. (2014). Mathematical Model of Stock Prices via a Fractional Brownian Motion Model with Adaptive Parameters.

Ton, D. (2004). Simulation of Fractional Brownian Motion, Thesis, University of Twente, Department of Mathematical Sciences, P.O. BOX 217, 7500 AE Enschede, Netherlands.

Wenyu, Zh. (2015). Introduction to Ito's Lemma, Lecture Note, Cornell University, Department of Statistical Sciences, May 6, 2015.

\section{Web of References}

https://www.stoodnt.com/blog/scopes-of-machine-learning-and-artificial-intelligence-in-banking-financialservices-ml-ai-the-future-of-fintechs/

https://medium.com/datadriveninvestor/neural-networks-activation-functions-e371202b56ff

https://missinglink.ai/guides/neural-network-concepts/7-types-neural-network-activation-functions-right/

http://www.datastuff.tech/machine-learning/why-do-neural-networks-need-an-activation-function/

https://medium.com/the-theory-of-everything/understanding-activation-functions-in-neural-networks-

$9491262884 \mathrm{e} 0$

https://www.youthkiawaaz.com/2019/07/future-of-artificial-intelligence-in-banks/

https://news.efinancialcareers.com/uk-en/328299/ai-in-trading-buy-side

https://ai.stackexchange.com/questions/7609/is-nassim-taleb-right-about-ai-not-being-able-to-accurately-predictcertain-type/7610

https://towardsdatascience.com/adam-latest-trends-in-deep-learning-optimization-6be9a291375c

The U.S. Hearing Youtube (Technology Companies \& Algorithms): https://www.youtube.com/watch?v=vtw4e68CkwU 
https://www.commerce.senate.gov/2019/6/optimizing-for-engagement-understanding-the-use-of-persuasivetechnology-on-internet-platforms

https://ai.stackexchange.com/questions/7088/how-to-choose-an-activation-function

https://mlfromscratch.com/activation-functions-explained/\#/

https://github.com/nadavo/mood.

\section{Backward propagation and Activation Functions}

$\mathrm{https}: / /$ www.youtube.com/watch?v=q555kfIFUCM

https://www.youtube.com/watch?v=-7scQpJT7uo

https://towardsdatascience.com/analyzing-different-types-of-activation-functions-in-neural-networks-which-oneto-prefer-e11649256209

http://vision.stanford.edu/teaching/cs231n-demos/linear-classify/

$\mathrm{http}: / /$ vision.stanford.edu/teaching/cs231n-demos/knn/

https://playground.tensorflow.org/\#activation=tanh\&batchSize=10\&dataset=circle\&regDataset=reg-

plane \&learningRate $=0.03$ \& regularizationRate $=0$ \&noise $=0$ \&networkShape $=4,2 \&$ seed $=0.41357 \&$ showTestData $=$ false $\&$ discretize $=$ false $\&$ percTrainData $=50 \& \mathrm{x}=$ true $\& \mathrm{y}=$ true $\& \mathrm{xTimes} Y=$ false $\& \mathrm{xSquared}=$ false $\& y$ Squared $=$ false $\& \cos \mathrm{X}=$ false $\& \sin \mathrm{X}=$ false $\& \cos \mathrm{Y}=$ false $\& \sin \mathrm{Y}=$ false $\& \operatorname{collectStats}=$ false \&problem $=$ classification \&initZero $=$ false \&hideText=false

\section{Appendix A}

\section{Research Companies and Data Sources}

This research paper was the extension of the Author's (Jamilu Auwalu Adamu (2015)) research findings published by the Asian Journal of Management Sciences, 03(08), 2015, 10-18 entitled "Banking and Economic Advanced Stressed Probability of Default Models". To statistically prove and explain to the general readers how the results of different types of Stressed SIGMOID and proposed Advanced Optimized Stochastic Activation Functions presented in this paper using financial industries DATA SET, the author uses the above research findings for illustration as repeating it by same author in this paper is an academic Tautology.

The author tested and implemented the models by considering FINANCIAL DATA of five (5) U.S. based companies listed on the platform of NEW YORK STOCK EXCHANGE (NYSE) from 2014 - 1991 (25 years) inclusive and extracted from Yahoo Finance as follows:
1) Microsoft Corporation (MSFT)
2) Exxon Mobil (XOM)
3) Chevron Corporation (CVX)
4) Honda Motor Corporation (HMC)
5) General Electric (GE)

\section{Other Selected Data Sources}

- Yahoo Finance

- Google Finance

- Federal Reserve Bank

- Economic Research

\section{Companies and Fundamental Macroeconomic Indicators used in the Research Work:}

In this research work, we consider:

a) Five (5) companies listed on the platform of New York Stock Exchange (NYSE) namely; Chevron Corporation, Honda Motor Corporation, Microsoft Corporation, Exxon Mobil Corporation, and General Electric Corporation for the period of Twenty Five (25) years $(1991$ - 2014) data

b) The stock returns of the five (5) companies under consideration

c) The U.S. GDP

d) The U.S. Inflation Rate 
e) The U.S. Prime Rate

f) The U.S. unemployment Rate

g) The U.S. USD/GBP Exchange Rate

h) The U.S. House Price

i) The U.S. Oil Price

j) The U.S. Gold Price

Simple Logistic Regression Model (SIGMOID or LOGIT):

$$
P D=\frac{1}{1+\exp \left(\sum_{i=0}^{K} \beta_{i} X_{i}\right)}
$$

PD is the probability of default. $X=\left(X_{1}, X_{2}, \ldots, X_{k}\right)$ is a vector of explanatory variables (Macro-economic Indicators).

The author disturbs it by introducing:

(i) Geometric Mean $\left(\mu_{A}\right)$ and Volatility $\left(\sigma_{A}\right)$ of the Macroeconomic Indicators used in the research work;

(ii) Geometric Mean $\left(\mu_{\text {Company }}\right)$ and Volatility $\left(\sigma_{\text {Company }}\right)$ of the Stock Return used in the research work; and

(iii) Probability Distribution Function $f\left(x ; \mu_{\text {company }}, \sigma_{\text {company }}, \xi\right)$.

As shown below:

Type A:

$$
P D_{\text {Stress }}=\frac{1}{1+\exp \mu_{A}\left(\sum_{i=0}^{K} \beta_{i} X_{i}\right) \mp \sigma_{A} f\left(x ; \mu_{\text {company }}, \sigma_{\text {company }}, \xi\right)}
$$

Type B:

$$
P D_{\text {Stress }}=\frac{1}{1+\exp \mu_{A}\left(\sum_{i=0}^{K} \beta_{i} X_{i}\right) \mp f\left(x ; \mu_{\text {company }}, \sigma_{\text {company }}, \xi\right)}
$$

Type C:

$$
P D_{\text {Stress }}=\frac{1}{1+\exp \left(\sum_{i=0}^{K} \beta_{i} X_{i}\right) \mp \sigma_{A} f\left(x ; \mu_{\text {company }}, \sigma_{\text {company }}, \xi\right)}
$$

Type D:

$$
P D_{\text {Stress }}=\frac{1}{1+\exp \left(\sum_{i=0}^{K} \beta_{i} X_{i}\right) \mp f\left(x ; \mu_{\text {company }}, \sigma_{\text {company }}, \xi\right)}
$$

\section{Stressed PROBIT:}

Type A: 


$$
P D_{\text {Stress }}=\Phi\left(\beta_{0}+\mu_{A} \sum_{j=1}^{J} \beta_{j} X_{j}\right) \pm \sigma_{A} f\left(x ; \mu_{\text {company }}, \sigma_{\text {company }}, \xi\right)
$$

Type B:

$$
P D_{\text {Stress }}=\Phi\left(\beta_{0}+\mu_{A} \sum_{j=1}^{J} \beta_{j} X_{j}\right) \pm f\left(x ; \mu_{\text {company }}, \sigma_{\text {company }}, \xi\right)
$$

Type C:

$$
P D_{\text {Stress }}=\Phi\left(\beta_{0}+\sum_{j=1}^{J} \beta_{j} X_{j}\right) \pm \sigma_{A} f\left(x ; \mu_{\text {company }}, \sigma_{\text {company }}, \xi\right)
$$

Type D:

$$
P D_{\text {Stress }}=\Phi\left(\beta_{0}+\sum_{j=1}^{J} \beta_{j} X_{j}\right) \pm f\left(x ; \mu_{\text {company }}, \sigma_{\text {company }}, \xi\right)
$$

Using Multiple Regression Analysis on the above Macroeconomic Indicators and QI Macros 2015 Software, we obtained the following components for determining the proposed Banking and Economic Advanced Stressed Probability of Default models for the five (5) companies under considerations.

Multiple Regression Model Component of CHEVRON Corporation (CVX) for calculating Probability of Default:

$$
\begin{aligned}
Y_{\text {CHEVRON }}= & 0.004+0.004 \times \Delta P(C H E V R O N)-0.199 \times \Delta P(G D P)+0.009 \times \Delta P(O I L)+0.009 \times \Delta P(I N F) \\
& -0.018 \times \Delta P(U E R)+0.002 \times \Delta P(G O L D)+0 \times \Delta P(I N T E R E S T)+0 \times \Delta P(U S D / G B P)
\end{aligned}
$$

Multiple Regression Model Component of GENERAL ELECTRIC(GE) for calculating Probability of Default:

$$
\begin{aligned}
Y_{G E}= & 0.004-0.001 \times \Delta P(G E)-0.207 \times \Delta P(G D P)+0.009 \times \Delta P(O I L)+0.016 \times \Delta P(I N F) \\
& -0.017 \times \Delta P(U E R)-0.001 \times \Delta P(G O L D)+0 \times \Delta P(I N T E R E S T)+0 \times \Delta P(U S D / G B P)
\end{aligned}
$$

Multiple Regression Model Component of MICROSOFT (MSFT) Corporation for calculating Probability of Default:

$$
\begin{aligned}
Y_{M S F T}= & 0.004-0.006 \times \Delta P(M S F T)-0.189 \times \Delta P(G D P)+0.009 \times \Delta P(O I L)+0.011 \times \Delta P(I N F) \\
& -0.017 \times \Delta P(U E R)+0.001 \times \Delta P(G O L D)+0 \times \Delta P(I N T E R E S T)+0 \times \Delta P(U S D / G B P)
\end{aligned}
$$

Multiple Regression Model Component of EXXON MOBIL (XOM) Corporation for calculating Probability of Default:

$$
\begin{aligned}
Y_{X O M}= & 0.004+0.002 \times \Delta P(X O M)-0.2 \times \Delta P(G D P)+0.009 \times \Delta P(O I L)+0.01 \times \Delta P(I N F) \\
& -0.018 \times \Delta P(U E R)+0.001 \times \Delta P(G O L D)+0 \times \Delta P(I N T E R E S T)+0 \times \Delta P(U S D / G B P)
\end{aligned}
$$

Multiple Regression Model Component of HONDA MOTOR CO., Ltd for calculating Probability of Default:

$$
\begin{aligned}
Y_{H O N D A}= & 0.004-0.004 \times \Delta P(H M C)-0.204 \times \Delta P(G D P)+0.009 \times \Delta P(O I L)+0.01 \times \Delta P(I N F) \\
& -0.018 \times \Delta P(U E R)+0.001 \times \Delta P(G O L D)+0 \times \Delta P(I N T E R E S T)+0 \times \Delta P(U S D / G B P)
\end{aligned}
$$


In this test of Goodness of fit, we consider Three (3) criteria:

(i) We accept if the Average of the ranks of Kolmogorov Smirnor, Anderson Darling and Chi-squared is less than or equal to Three (3)

(ii) At least Two (2) probability distributions must included in the selection

(iii) We select the most occur probability distribution as the qualify candidate in each case of test of goodness of fit of the stock returns as follows

\section{LOG - LOGISTIC (3P) PROBABILITY DISTRIBUTION}

$$
f(x ; \mu, \sigma, \xi)=\left\{\begin{array}{c}
\frac{\left(1+\frac{\xi(x-\mu)}{\sigma}\right)^{-(1 / \xi+1)}}{\left[1+\left(1+\frac{\xi(x-\mu)^{-1 / \xi}}{\sigma}\right)\right]^{2}} ; x \geq \mu \\
0 \quad ; \quad \text { elsewhere }(x \leq \mu)
\end{array}\right.
$$

is called Generalized Log-Logistic or Log-Logistic (3P) Probability Distribution. Where, $\mu \in \square \quad$ is the location parameter, $\sigma>0$ the scale parameter and $\xi \in \square$ the shape parameter. The shape parameter $\xi$ is often restricted to lie in $[-1,1]$, when the probability density function is bounded.

However, in this research work we will restrict $\xi$ to three cases only $\xi=1$. However, one can test, for $\xi=0$ or $\xi=-1$ in the subsequent researchers.

\section{Appendix B}

\section{Statistical Data Analysis}

\section{SUMMARY RESULT OF TEST OF GOODNESS OF FIT OF CHEVRON CORPORATION STOCK RETURN}

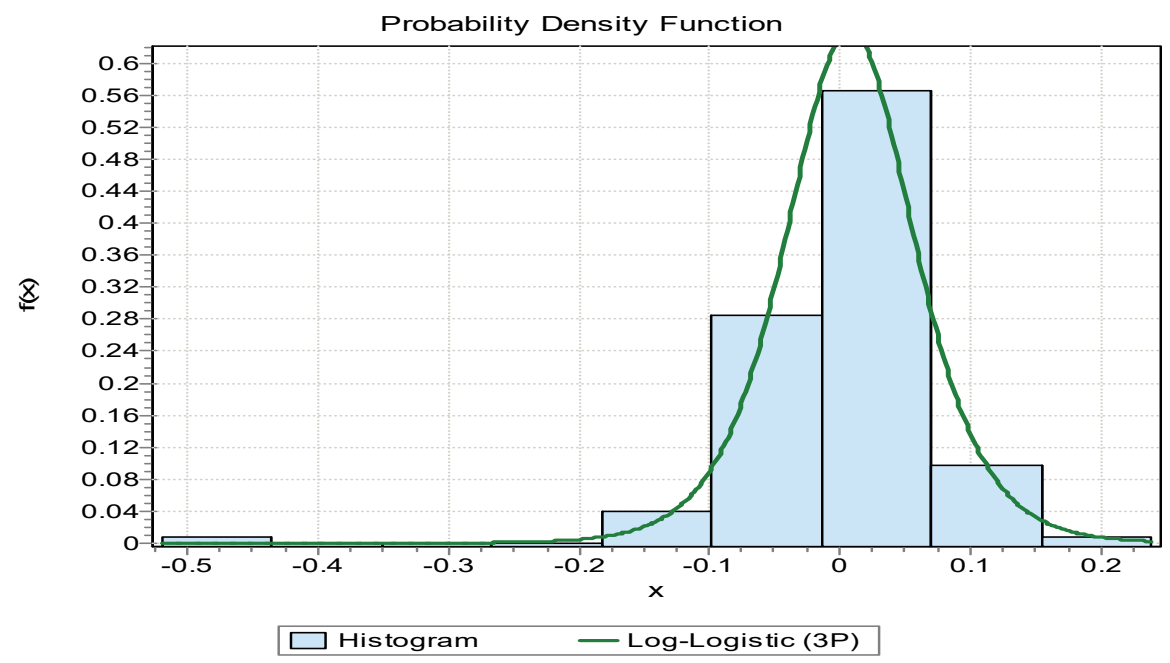




\begin{tabular}{|l|l|l|l|l|}
\hline $\begin{array}{l}\text { Name of Probability } \\
\text { Distribution }\end{array}$ & $\begin{array}{l}\text { Kolmogorov } \\
\text { Smirnov Rank }\end{array}$ & $\begin{array}{l}\text { Anderson Darling } \\
\text { Rank }\end{array}$ & Chi- Squared Rank & Average \\
\hline Burr & 3 & 3 & 2 & 2.07 \\
\hline Dagum & 2 & 1 & 1 & 1.33 \\
\hline Log - Logistic(3P) & 1 & 2 & 3 & 2.0 \\
\hline
\end{tabular}

\section{Chevron Calculated Parameters:}

Using our data sources, Microsoft EXCEL and QI Macros 2015, we obtained the Chevron calculated parameters as follows:

Geometric Mean and Volatility (Standard Deviation) of the Macroeconomic indicators used in the case of Chevron are given by:

$\mu_{\text {GEO }}($ Chevron $)=0.030383975$

$\sigma_{G E O}($ Chevron $)=0.111414539$

Geometric Mean and Volatility (Standard Deviation) of Chevron Stock Return are given by:

$\mu_{\text {STOCK }}($ Chevron $)=0.004402791$

$\sigma_{\text {STOCK }}($ Chevron $)=0.06909299$

\section{Results of Stressed SIGMOID and Probit MODELS for Chevron Corporation:}

Under Chevron Corporation, on the month of June, 2014, the probability of default using the existing Sigmoid is $0.499097747 \%$ and that of probit is $0.501439786 \%$, whereas, using Stressed Sigmoid and Probit models are: $0.499976914 \%, 0.499968258 \%, 0.500011436 \%, 0.499933742 \%, 0.49910206 \%, 0.499093434 \%, 0.499136461 \%$, $0.499059039 \%, 0.501573711 \%, 0.50160834 \%, 0.501435622 \%, 0.501746429 \%, 0.501422471 \%, 0.5014571 \%$, $0.501284382 \%$, and $0.501595189 \%$ respectively. Generally, this can be seen in the table below:

\begin{tabular}{|c|c|c|c|c|c|c|c|c|c|c|c|c|c|c|c|c|c|c|}
\hline & M1 & M1 & M1 & M1 & M1 & M1 & M1 & M1 & & & M2 & M2 & M2 & M2 & M2 & M2 & M2 & \\
\hline & TYPE & TYPE & TYPE & TYPE & TYPE & TYPE & TYPE & TYPE & & PROBI & TYPE & TYPE & TYPE & TYPE & TYPE & TYPE & TYPE & M2 TYPE \\
\hline Date & A- & $\mathrm{A}^{+}$ & B- & $\mathrm{B}+$ & C- & $\mathrm{C}+$ & D- & D+ & LOGIT & $\mathrm{T}$ & A. & $\mathrm{A}^{+}$ & B- & B+ & C- & $\mathrm{C}+$ & D. & D+ \\
\hline $01 / 12 / 20$ & 0.4999 & 0.49996 & 0.4999 & 0.49995 & 0.4989 & 0.49896 & 0.4989 & 0.49895 & 0.4989 & 0.5016 & 0.5015 & 0.50160 & 0.5015 & 0.50167 & 0.5016 & 0.50165 & 0.5015 & 0.501715 \\
\hline 14 & 70779 & 6686 & 87104 & 0363 & 72963 & 8885 & 89222 & 2627 & 70924 & 42165 & 88987 & 5363 & 23683 & 0667 & 33977 & 0353 & 68673 & 657 \\
\hline $03 / 11 / 20$ & 0.4999 & 0.49998 & 0.4999 & 0.49998 & 0.4996 & 0.49960 & 0.4996 & 0.49960 & 0.4996 & 0.5006 & 0.5015 & 0.50156 & 0.5015 & 0.50156 & 0.5006 & 0.50062 & 0.5006 & 0.500629 \\
\hline 14 & 88014 & 8014 & 88014 & 8014 & 055 & 55 & 055 & 55 & 055 & 29531 & 66407 & 6407 & 66407 & 6407 & 29531 & 9531 & 29531 & 531 \\
\hline $01 / 10 / 20$ & 0.4999 & 0.49999 & 0.4999 & 0.49999 & 0.4999 & 0.49992 & 0.4999 & 0.49992 & 0.4999 & 0.5001 & 0.5015 & 0.50155 & 0.5015 & 0.50155 & 0.5001 & 0.50012 & 0.5001 & 0.500126 \\
\hline 14 & 97599 & 7596 & 9761 & 7584 & 20916 & 0913 & 20928 & 0902 & 20915 & 26202 & 51108 & 112 & 51061 & 1167 & 26196 & 6208 & 26149 & 254 \\
\hline $02 / 09 / 20$ & 0.4999 & 0.49998 & 0.4999 & 0.49998 & 0.4994 & 0.49941 & 0.4994 & 0.49941 & 0.4994 & 0.5009 & 0.5015 & 0.50157 & 0.5015 & 0.50157 & 0.5009 & 0.50092 & 0.5009 & 0.500929 \\
\hline 14 & 82294 & 2294 & 82294 & 2294 & 17245 & 7245 & 17245 & 7245 & 17245 & 29942 & 75535 & 5535 & 75535 & 5535 & 29942 & 9942 & 29942 & 942 \\
\hline $01 / 08 / 20$ & 0.4999 & 0.49998 & 0.4999 & 0.49998 & 0.4993 & 0.49939 & 0.4993 & 0.49939 & 0.4993 & 0.5009 & 0.5015 & 0.50157 & 0.5015 & 0.50157 & 0.5009 & 0.50096 & 0.5009 & 0.500970 \\
\hline 14 & 81556 & 1525 & 81677 & 1404 & 92475 & 2445 & 92596 & 2323 & 9246 & 69493 & 76675 & 6797 & 76189 & 7284 & 69432 & 9554 & 68946 & 041 \\
\hline $01 / 07 / 20$ & 0.4999 & 0.49999 & 0.4999 & 0.49999 & 0.4998 & 0.49985 & 0.4998 & 0.49984 & 0.4998 & 0.5002 & 0.5015 & 0.50155 & 0.5015 & 0.50157 & 0.5002 & 0.50023 & 0.5002 & 0.500252 \\
\hline 14 & 9598 & 5049 & 9969 & 1338 & 52831 & 1901 & 56539 & 8192 & 52366 & 3559 & 52576 & 6299 & 37734 & 1141 & 33729 & 7451 & 18886 & 294 \\
\hline $02 / 06 / 20$ & 0.4999 & 0.49996 & 0.5000 & 0.49993 & 0.4991 & 0.49909 & 0.4991 & 0.49905 & 0.4990 & 0.5014 & 0.5015 & 0.50160 & 0.5014 & 0.50174 & 0.5014 & 0.50145 & 0.5012 & 0.501595 \\
\hline 14 & 76914 & 8258 & 11436 & 3742 & 0206 & 3434 & 36461 & 9039 & 97747 & 39786 & 73711 & 834 & 35622 & 6429 & 22471 & 71 & 84382 & 189 \\
\hline $01 / 05 / 20$ & 0.4999 & 0.49997 & 0.4999 & 0.49996 & 0.4992 & 0.49927 & 0.4992 & 0.49925 & 0.4992 & 0.5011 & 0.5015 & 0.50158 & 0.5015 & 0.50164 & 0.5011 & 0.50116 & 0.5010 & 0.501221 \\
\hline 14 & 7971 & 6176 & 93802 & 2085 & 75826 & 2302 & 89878 & 825 & 74064 & 58426 & 75409 & 9545 & 19036 & 5917 & 51358 & 5494 & 94985 & 867 \\
\hline $01 / 04 / 20$ & 0.4999 & 0.49996 & 0.4999 & 0.49993 & 0.4989 & 0.49889 & 0.4989 & 0.49886 & 0.4988 & 0.5017 & 0.5015 & 0.50161 & 0.5014 & 0.50172 & 0.5017 & 0.50177 & 0.5016 & 0.501881 \\
\hline 14 & 69966 & 3096 & 97362 & 5704 & 01885 & 5045 & 29164 & 7769 & 98465 & 57793 & 86947 & 4429 & 77356 & 402 & 44052 & 1534 & 34462 & 125 \\
\hline
\end{tabular}




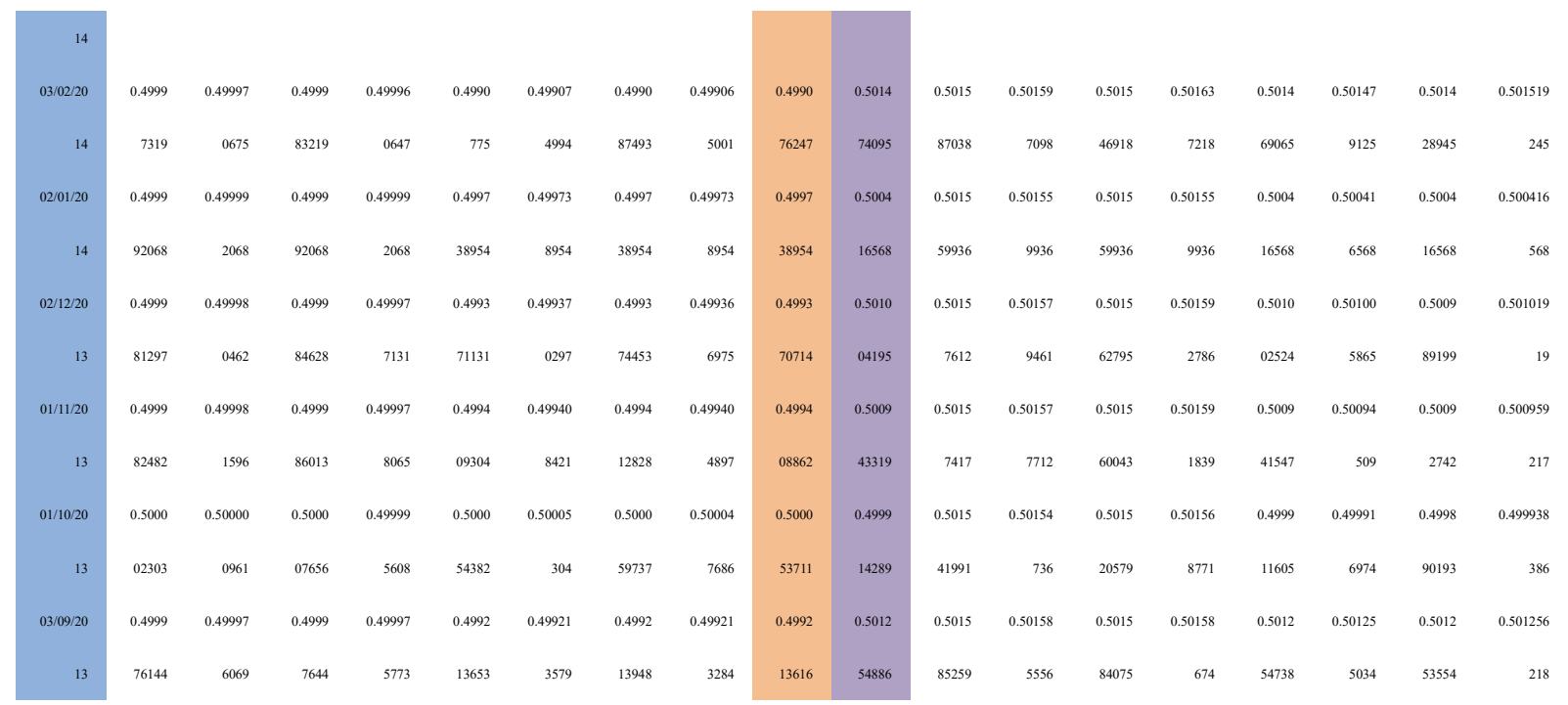

Source: Jamilu Auwalu Adamu (2015), Asian Journal of Management Sciences, 03(08), 2015, 10-18

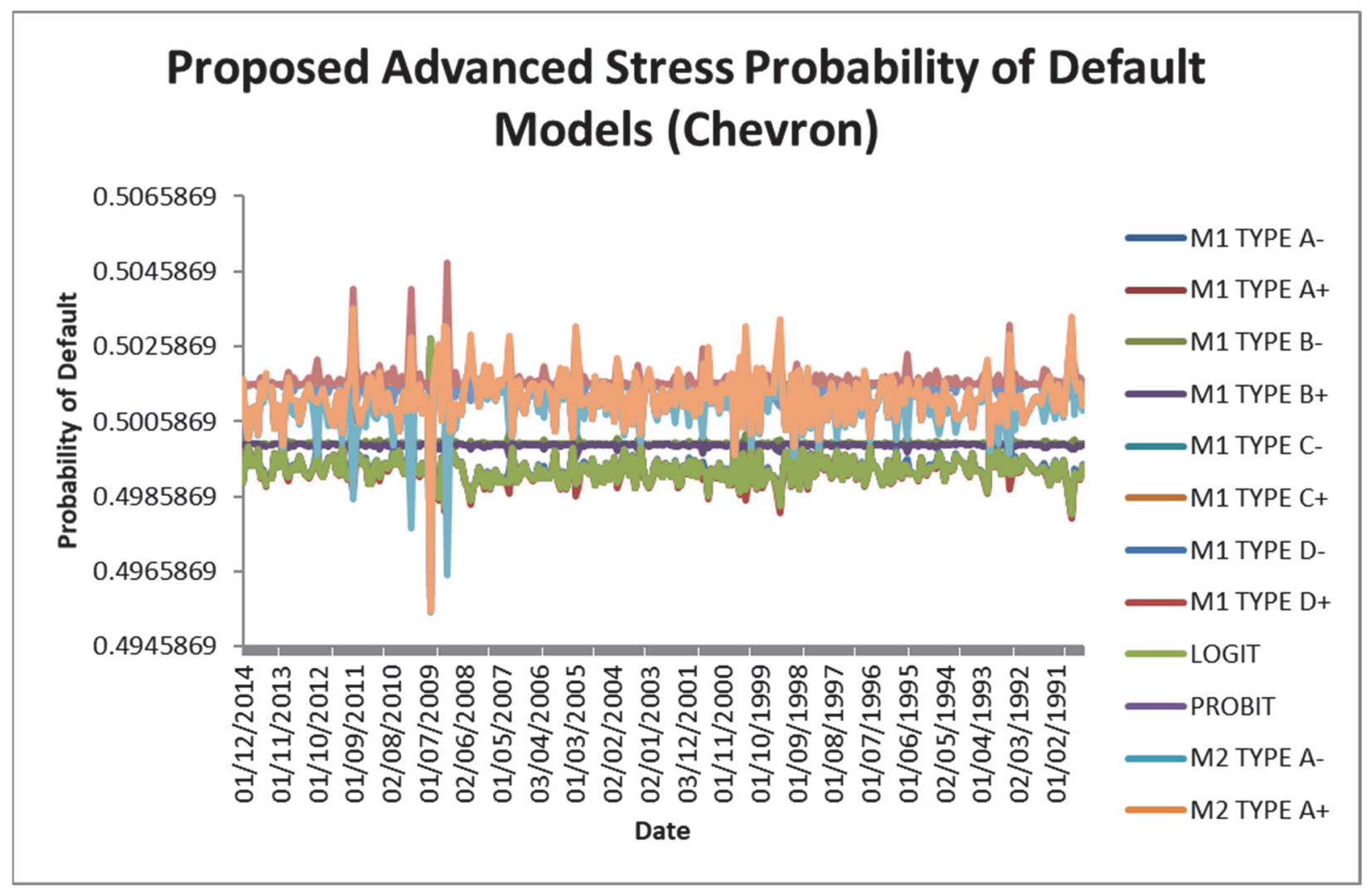

SUMMARY RESULT OF TEST OF GOODNESS OF FIT OF HONDA MOTOR CORPORATION STOCK RETURN 


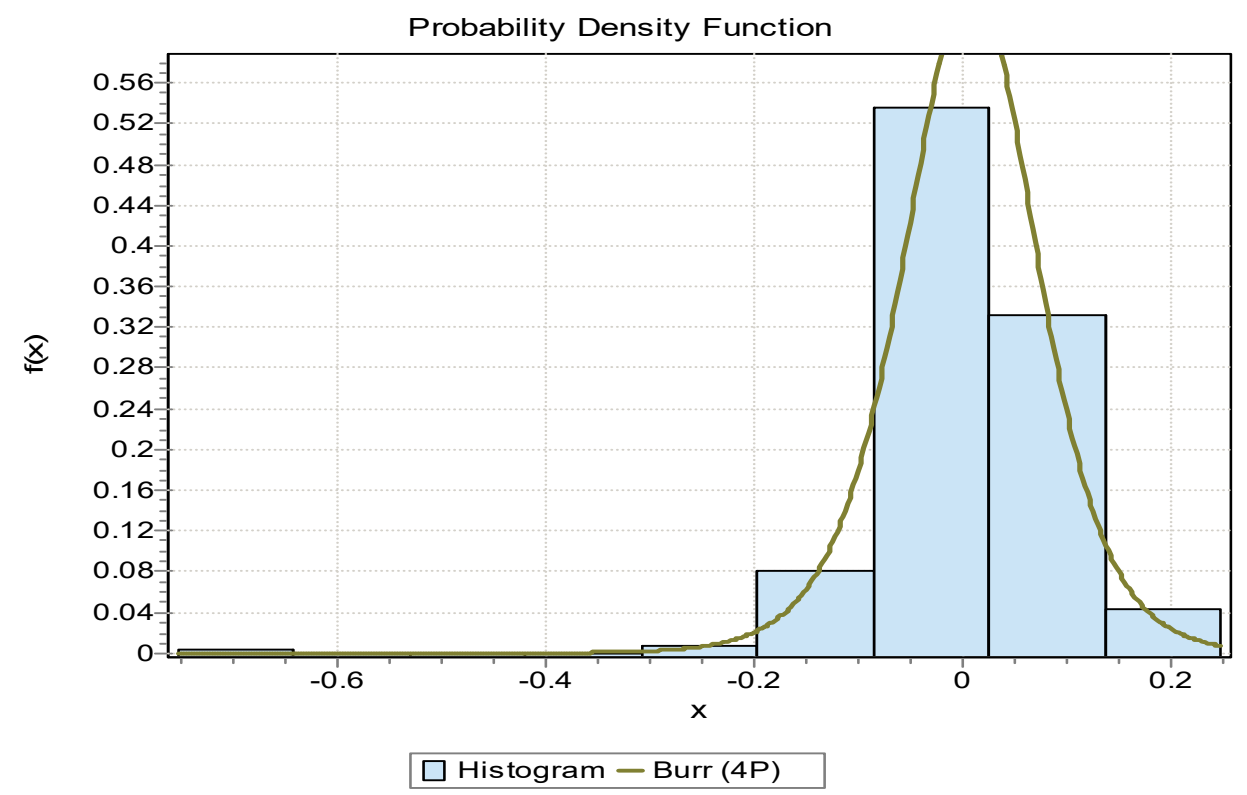

\begin{tabular}{|l|l|l|l|l|}
\hline $\begin{array}{l}\text { Name of Probability } \\
\text { Distribution }\end{array}$ & $\begin{array}{l}\text { Kolmogorov } \\
\text { Smirnov Rank }\end{array}$ & $\begin{array}{l}\text { Anderson Darling } \\
\text { Rank }\end{array}$ & Chi- Squared Rank & Average \\
\hline Burr & 1 & 2 & 1 & 1.33 \\
\hline Dagum & 2 & 3 & 2 & 2.33 \\
\hline Log - Logistic(3P) & 3 & 1 & 3 & 2.33 \\
\hline
\end{tabular}

Geometric Mean and Volatility (Standard Deviation) of the Macroeconomic indicators used in the case of Honda Motor are given by:

$\mu_{\text {GEO }}($ Honda $)=0.031352397$

$\sigma_{G E O}($ Honda $)=0.114001187$

Geometric Mean and Volatility (Standard Deviation) of Honda Motor Stock Return are given by:

$\mu_{\text {STOCK }}($ Honda $)=0.005839335$

$\sigma_{\text {STOCK }}($ Honda $)=0.084945727$

\section{Results of Stressed SIGMOID and Probit MODELS for Honda Motor Corporation:}

Under Honda Motor, on the month of December, 2014, the probability of default using the existing Sigmoid is $0.49898654 \%$ and that of probit is $0.501617235 \%$, whereas, using Stressed Sigmoid and Probit models are: $0.49996827 \%, 0.499968182 \%, 0.49996784 \%, 0.49896591 \%, 0.498986503 \%, 0.498986932 \%, 0.498986163 \%$, $0.501594751 \%, 0.501595103 \%, 0.501593383 \%, 0.50159647 \%, 0.501617059 \%, 0.50161741 \%, 0.501615691 \%$, and $0.501618778 \%$ respectively..This can be seen below:

\begin{tabular}{|c|c|c|c|c|c|c|c|c|c|c|c|c|c|c|c|c|c|c|}
\hline & M1 & M1 & M1 & M1 & M1 & M1 & M1 & M1 & & & M2 & M2 & M2 & M2 & M2 & M2 & M2 & M2 \\
\hline & TYPE & TYPE & TYPE & TYPE & TYPE & TYPE & TYPE & TYPE & & PROBI & TYPE & TYPE & TYPE & TYPE & TYPE & TYPE & TYPE & TYPE \\
\hline Date & A- & $\mathrm{A}^{+}$ & B- & B+ & C- & C+ & D- & D+ & LOGIT & $\mathrm{T}$ & A- & $\mathrm{A}^{+}$ & B- & B + & C- & $\mathrm{C}^{+}$ & D- & D+ \\
\hline $01 / 12 /$ & 0.4999 & 0.49996 & 0.4999 & 0.49996 & 0.4989 & 0.49898 & 0.4989 & 0.49898 & 0.4989 & 0.5016 & 0.5015 & 0.50159 & 0.5015 & 0.50159 & 0.5016 & 0.50161 & 0.5016 & 0.50161 \\
\hline 2014 & 6827 & 8182 & 68612 & 784 & 86591 & 6503 & 86932 & 6163 & 86547 & 17235 & 94751 & 5103 & 93383 & 647 & 17059 & 741 & 15691 & 8778 \\
\hline $03 / 11 /$ & 0.5000 & 0.49996 & 0.5001 & 0.49980 & 0.4994 & 0.49942 & 0.4996 & 0.49926 & 0.4994 & 0.5008 & 0.5015 & 0.50170 & 0.5009 & 0.50234 & 0.5008 & 0.50097 & 0.5001 & 0.50160 \\
\hline 2014 & 02916 & 2034 & 6184 & 3237 & 61403 & 061 & 19983 & 2157 & 41006 & 92025 & 41668 & 5204 & 06182 & 069 & 10258 & 3793 & 74771 & 928 \\
\hline $01 / 10$ & 0.5000 & 0.49996 & 0.5002 & 0.49972 & 0.4998 & 0.49983 & 0.5001 & 0.49959 & 0.4998 & 0.5002 & 0.5015 & 0.50176 & 0.5005 & 0.50272 & 0.5000 & 0.50033 & 0.4991 & 0.50129 \\
\hline 2014 & 26642 & 5058 & 66096 & 5893 & 98359 & 6807 & 3769 & 7764 & 67581 & 1131 & 22306 & 8645 & 65052 & 5899 & 8814 & 4479 & 30886 & 1733 \\
\hline
\end{tabular}




\begin{tabular}{|c|c|c|c|c|c|c|c|c|c|c|c|c|c|c|c|c|c|c|}
\hline $02 / 09 /$ & 0.4999 & 0.49997 & 0.4999 & 0.49997 & 0.4993 & 0.49933 & 0.4993 & 0.49933 & 0.4993 & 0.5010 & 0.5016 & 0.50161 & 0.5016 & 0.50161 & 0.5010 & 0.50106 & 0.5010 & 0.50106 \\
\hline 2014 & 79001 & 8997 & 79016 & 8981 & 30153 & 0149 & 30169 & 0134 & 30151 & 68923 & 11599 & 1615 & 11536 & 1677 & 68915 & 8931 & 68852 & 8994 \\
\hline $01 / 08 /$ & 0.4999 & 0.49997 & 0.5000 & 0.49995 & 0.4993 & 0.49936 & 0.4993 & 0.49933 & 0.4993 & 0.5010 & 0.5016 & 0.50162 & 0.5015 & 0.50171 & 0.5010 & 0.50102 & 0.5009 & 0.50111 \\
\hline 2014 & 82846 & 722 & 04707 & 5362 & 65952 & 034 & 87759 & 8535 & 63146 & 16271 & 05063 & 7566 & 17616 & 5013 & 0502 & 7523 & 17573 & 497 \\
\hline $01 / 07 /$ & 0.4999 & 0.49999 & 0.4999 & 0.49999 & 0.4998 & 0.49985 & 0.4998 & 0.49985 & 0.4998 & 0.5002 & 0.5016 & 0.50163 & 0.5016 & 0.50164 & 0.5002 & 0.50023 & 0.5002 & 0.50023 \\
\hline 2014 & 957 & 5279 & 97338 & 3642 & 5635 & 5929 & 57986 & 4292 & 56139 & 29569 & 3807 & 9755 & 31521 & 6305 & 28726 & 0411 & 22176 & 6961 \\
\hline $02 / 06 /$ & 0.4999 & 0.49997 & 0.4999 & 0.49997 & 0.4991 & 0.49914 & 0.4991 & 0.49914 & 0.4991 & 0.5013 & 0.5016 & 0.50160 & 0.5015 & 0.50161 & 0.5013 & 0.50136 & 0.5013 & 0.50137 \\
\hline 2014 & 7364 & 2967 & 76255 & 0352 & 4883 & 8159 & 51436 & 5552 & 48494 & 58805 & 02388 & 5081 & 91927 & 5542 & 57459 & 0151 & 46998 & 0613 \\
\hline $01 / 05 /$ & 0.4999 & 0.49997 & 0.5000 & 0.49993 & 0.4993 & 0.49929 & 0.4993 & 0.49926 & 0.4992 & 0.5011 & 0.5015 & 0.50162 & 0.5014 & 0.50175 & 0.5011 & 0.50113 & 0.5009 & 0.50127 \\
\hline 2014 & 82452 & 3628 & 16742 & 9343 & 03967 & 5167 & 38164 & 0975 & 99567 & 17729 & 87455 & 2752 & 50293 & 9914 & 0008 & 5378 & 62919 & 254 \\
\hline $01 / 04 /$ & 0.4999 & 0.49994 & 0.5001 & 0.49976 & 0.4988 & 0.49885 & 0.4990 & 0.49868 & 0.4988 & 0.5017 & 0.5015 & 0.50168 & 0.5008 & 0.50238 & 0.5017 & 0.50188 & 0.5010 & 0.50258 \\
\hline 2014 & 87283 & 2305 & 62143 & 7598 & 99443 & 466 & 73543 & 0713 & 77051 & 91965 & 0542 & 5359 & 06189 & 459 & 01995 & 1935 & 02765 & 1165 \\
\hline $03 / 03 /$ & & & & & & & & & & & & & & & & & & \\
\hline 2014 & & & & & & & & & & & & & & & & & & \\
\hline $03 / 02 /$ & 0.4999 & 0.49996 & 0.5000 & 0.49990 & 0.4990 & 0.49906 & 0.4991 & 0.49900 & 0.4990 & 0.5014 & 0.5015 & 0.50163 & 0.5013 & 0.50187 & 0.5014 & 0.50150 & 0.5011 & 0.50174 \\
\hline 2014 & 78844 & 3263 & 39398 & 2728 & 84505 & 898 & 44843 & 8661 & 76742 & 73304 & 72334 & 4664 & 30127 & 6871 & 4214 & 4469 & 99932 & 6677 \\
\hline $02 / 01 /$ & 0.4999 & 0.49998 & 0.4999 & 0.49998 & 0.4995 & 0.49955 & 0.4995 & 0.49955 & 0.4995 & 0.5007 & 0.5016 & 0.50163 & 0.5016 & 0.50163 & 0.5007 & 0.50070 & 0.5007 & 0.50070 \\
\hline 2014 & 86114 & 6114 & 86114 & 6114 & 57102 & 7102 & 57102 & 7102 & 57102 & 06763 & 32929 & 2929 & 32929 & 2929 & 06763 & 6763 & 06763 & 6763 \\
\hline $02 / 12 /$ & 0.4999 & 0.49997 & 0.5000 & 0.49995 & 0.4993 & 0.49935 & 0.4993 & 0.49933 & 0.4993 & 0.5010 & 0.5016 & 0.50162 & 0.5015 & 0.50171 & 0.5010 & 0.50103 & 0.5009 & 0.50112 \\
\hline 2013 & 8262 & 6974 & 04563 & 5033 & 58429 & 2796 & 80317 & 0911 & 55612 & 28293 & 04648 & 7236 & 16875 & 5009 & 16999 & 9587 & 29226 & 7361 \\
\hline $01 / 11 /$ & 0.4999 & 0.49997 & 0.5000 & 0.49993 & 0.4994 & 0.49946 & 0.4995 & 0.49942 & 0.4994 & 0.5008 & 0.5015 & 0.50163 & 0.5014 & 0.50178 & 0.5008 & 0.50086 & 0.5006 & 0.50101 \\
\hline 2013 & 88346 & 8427 & 26895 & 9885 & 7504 & 5141 & 1351 & 6679 & 7009 & 45613 & 93434 & 3114 & 39243 & 7305 & 25773 & 5453 & 71582 & 9644 \\
\hline $01 / 10$ & 0.5000 & 0.49999 & 0.5000 & 0.49997 & 0.5000 & 0.50008 & 0.5001 & 0.50006 & 0.5000 & 0.4998 & 0.5016 & 0.50165 & 0.5015 & 0.50175 & 0.4998 & 0.49987 & 0.4997 & 0.49997 \\
\hline 2013 & 05942 & 9583 & 30655 & 4873 & 91295 & 4934 & 16016 & 0215 & 88114 & 5939 & 32711 & 8147 & 33867 & 6992 & 46672 & 2108 & 47827 & 0953 \\
\hline $03 / 09 /$ & 0.4999 & 0.49997 & 0.5000 & 0.49993 & 0.4993 & 0.49929 & 0.4993 & 0.49925 & 0.4992 & 0.5011 & 0.5015 & 0.50162 & 0.5014 & 0.50178 & 0.5011 & 0.50114 & 0.5009 & 0.50130 \\
\hline 2013 & 34 & 2806 & 2303 & 2869 & 01673 & 1423 & 41509 & 1594 & 96548 & 22547 & 83918 & 5035 & 24139 & 4814 & 01988 & 3105 & 42209 & 2884 \\
\hline
\end{tabular}

Source: Jamilu Auwalu Adamu (2015), Asian Journal of Management Sciences, 03(08), 2015, 10-18 


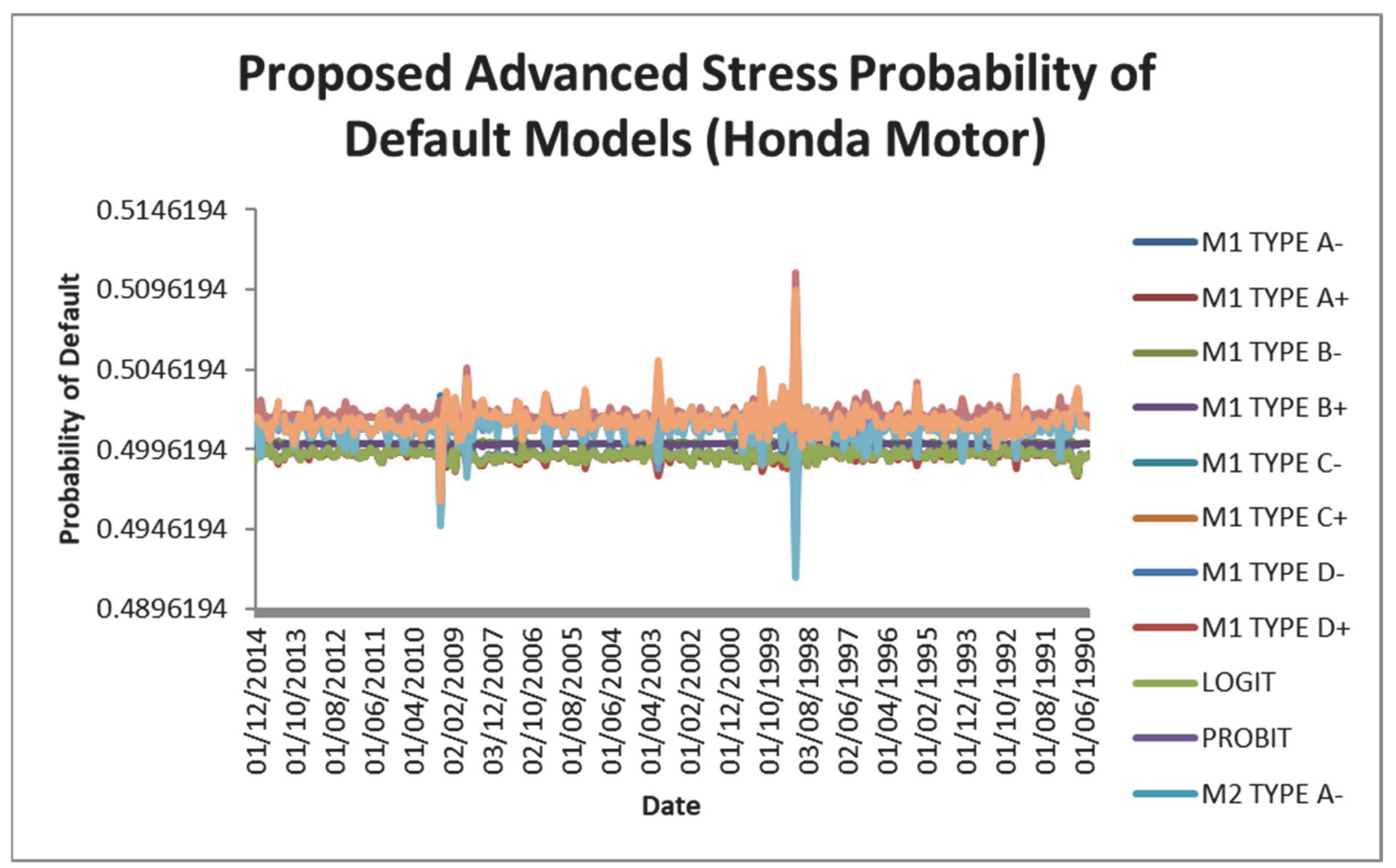

\section{SUMMARY RESULT OF TEST OF GOODNESS OF FIT OF MICROSOFT CORPORATION STOCK RETURN}

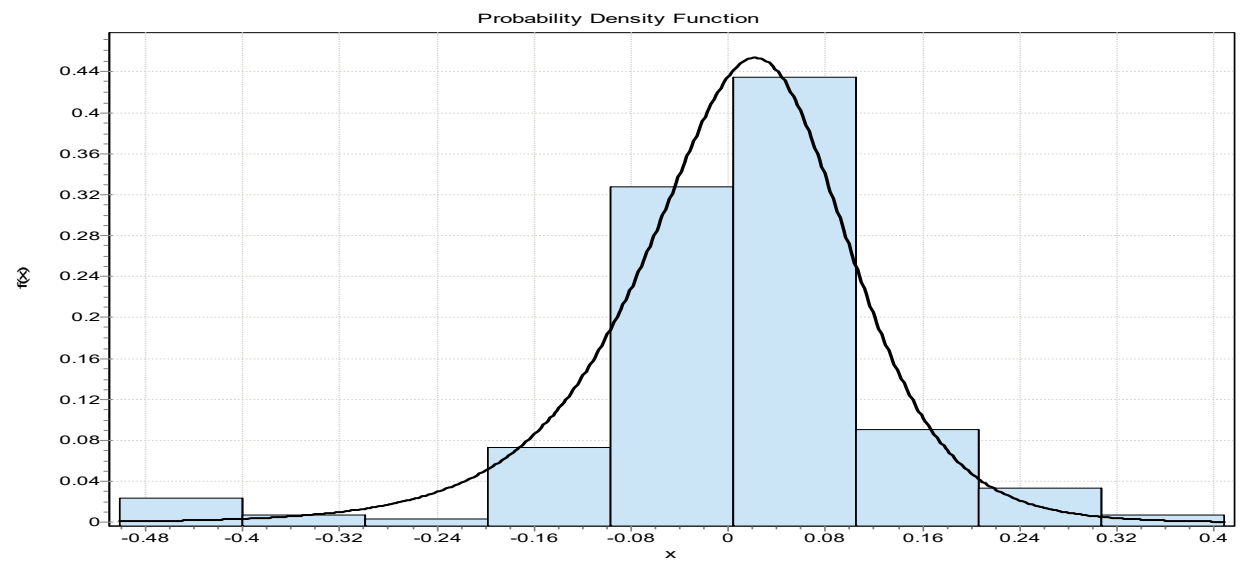

$\square$ Histogram -Dagum (4P)

\begin{tabular}{|l|l|l|l|l|}
\hline $\begin{array}{l}\text { Name of Probability } \\
\text { Distribution }\end{array}$ & $\begin{array}{l}\text { Kolmogorov } \\
\text { Smirnov Rank }\end{array}$ & $\begin{array}{l}\text { Anderson Darling } \\
\text { Rank }\end{array}$ & Chi- Squared Rank & Average \\
\hline Dagum & 1 & 1 & 1 & 1.0 \\
\hline Log - Logistic(3P) & 2 & 4 & 4 & 3.33 \\
\hline
\end{tabular}

Geometric Mean and Volatility (Standard Deviation) of the Macroeconomic indicators used in the case of Microsoft Corporation are given by:

$\mu_{G E O}(M S F T)=0.031352397$

$\sigma_{G E O}(M S F T)=0.117906073$

Geometric Mean and Volatility (Standard Deviation) of Microsoft Corporation Stock Return are given by: 
$\mu_{\text {STOCK }}(M S F T)=0.006798657$
$\sigma_{\text {STOCK }}(M S F T)=0.115022493$

Results of Stressed SIGMOID and Probit MODELS for Microsoft Corporation:

Under Microsoft Corporation, on the month of December, 2014, the probability of default using the existing Simoid is $0.499506722 \%$ and that of probit is $0.500787158 \%$, whereas, the Stressed Sigmoid and Probit models are: $\quad 0.499984689 \%, \quad 0.499983853 \%, \quad 0.499987817 \%, \quad 0.499980725 \%, \quad 0.499507139 \%, \quad 0.499506304 \%$, $0.49951026 \%, 0.499503183 \%, 0.50161708 \%, 0.501620425 \%, 0.501604569 \%, 0.501632936 \%, 0.500785486 \%$, $0.50078831 \%$, and $0.500772975 \%, 0.500801342 \%$ respectively. This can be seen as follows:

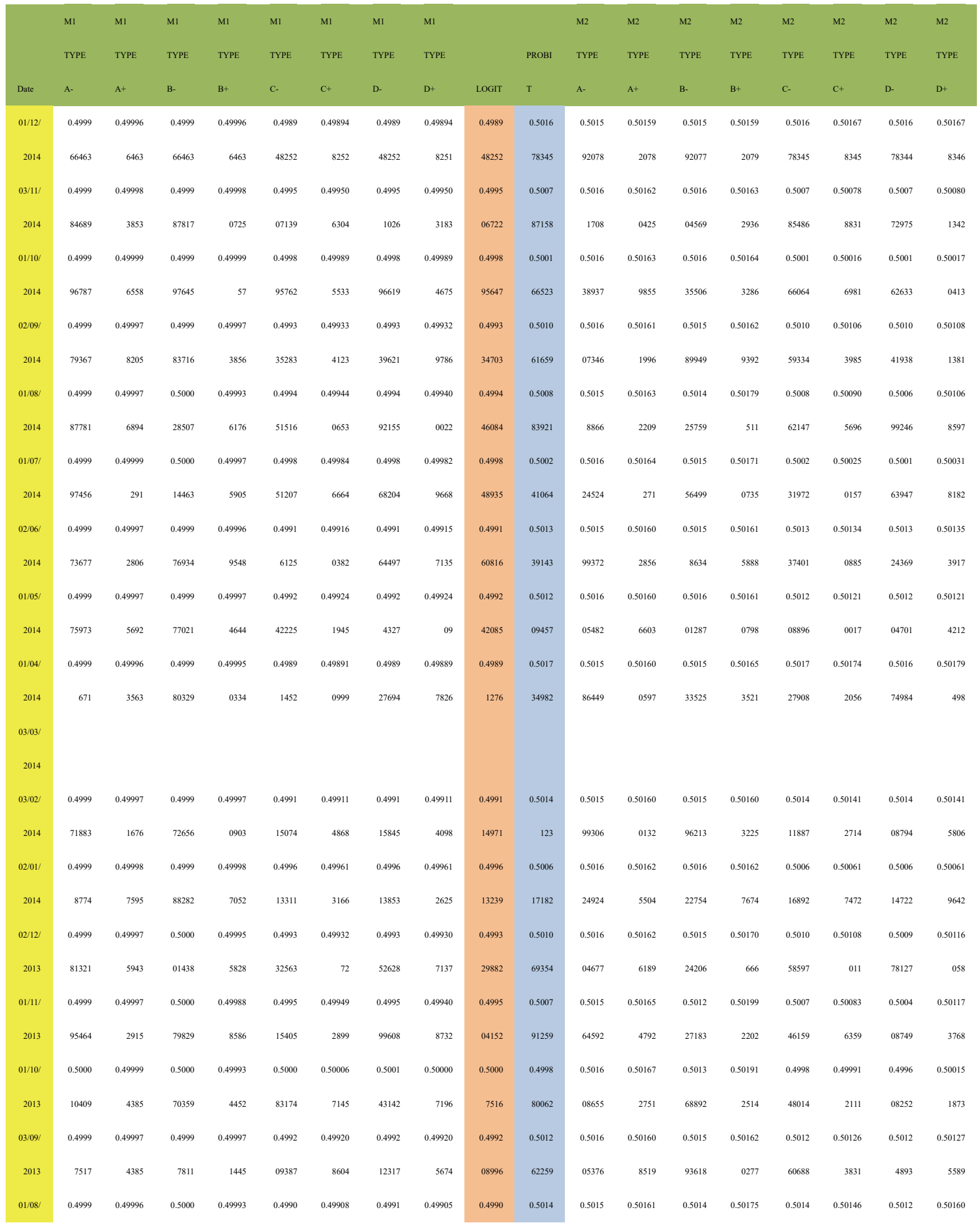




$$
\begin{array}{rrrrrrrrrrrrrrrrrrr}
2013 & 75756 & 6337 & 10991 & 1108 & 96682 & 7297 & 31794 & 2191 & 9199 & 48974 & 74127 & 1806 & 33181 & 2752 & 30134 & 7813 & 89188 & 8759 \\
01107 / & 0.5000 & 0.49991 & 0.5004 & 0.49948 & 0.4993 & 0.49919 & 0.4997 & 0.49876 & 0.4992 & 0.5011 & 0.5013 & 0.50185 & 0.4996 & 0.50360 & 0.5009 & 0.50142 & 0.4992 & 0.50317 \\
2013 & 34658 & 7798 & 72277 & 1148 & 12548 & 6026 & 48904 & 0635 & 5428 & 89996 & 86894 & 4379 & 3819 & 3082 & 56253 & 3738 & 0755 & 2442
\end{array}
$$

Source: Jamilu Auwalu Adamu (2015), Asian Journal of Management Sciences, 03(08), 2015, 10-18

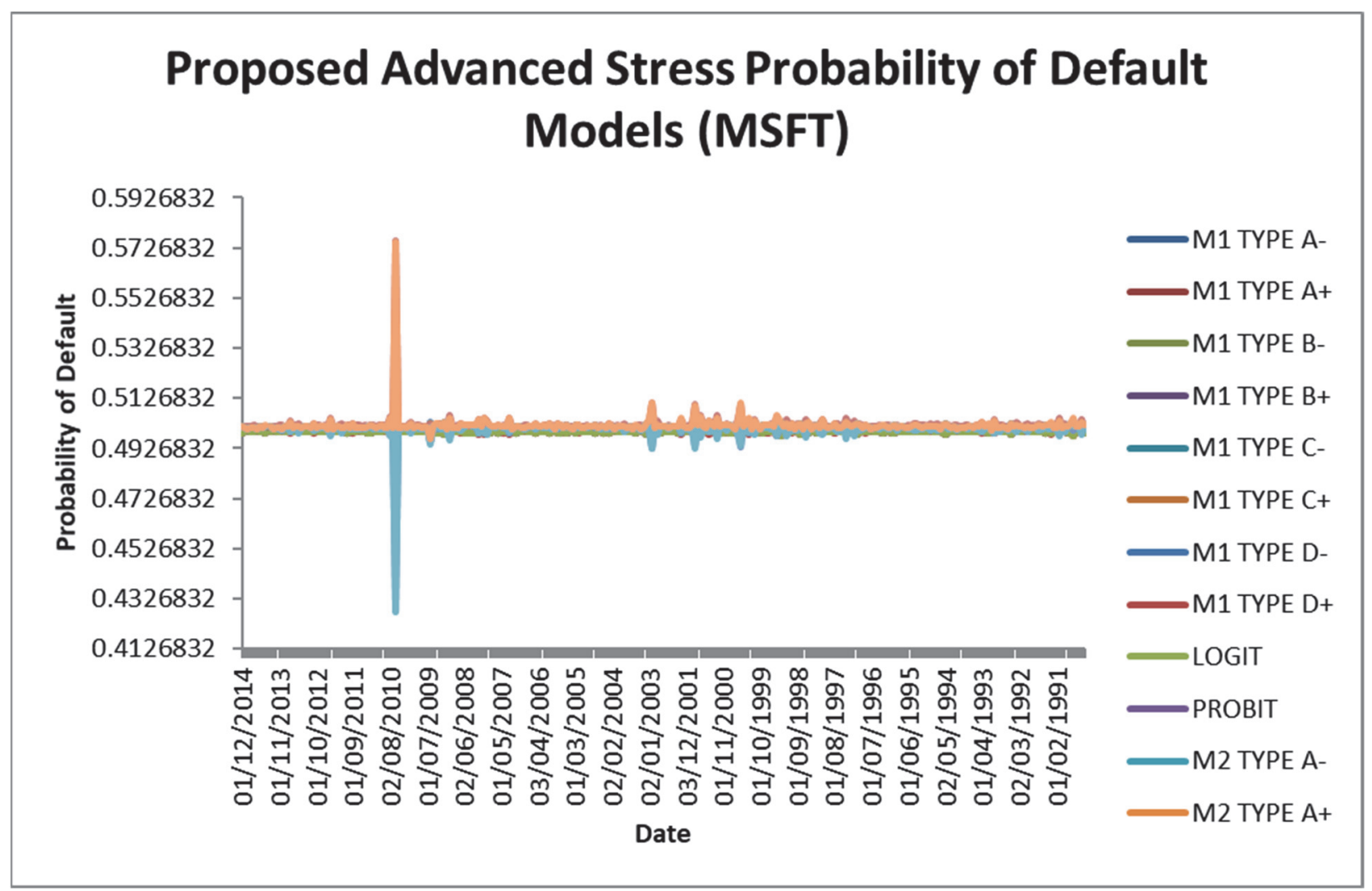

\section{SUMMARY RESULT OF TEST OF GOODNESS OF FIT OF EXXON MOBIL CORPORATION} STOCK RETURN

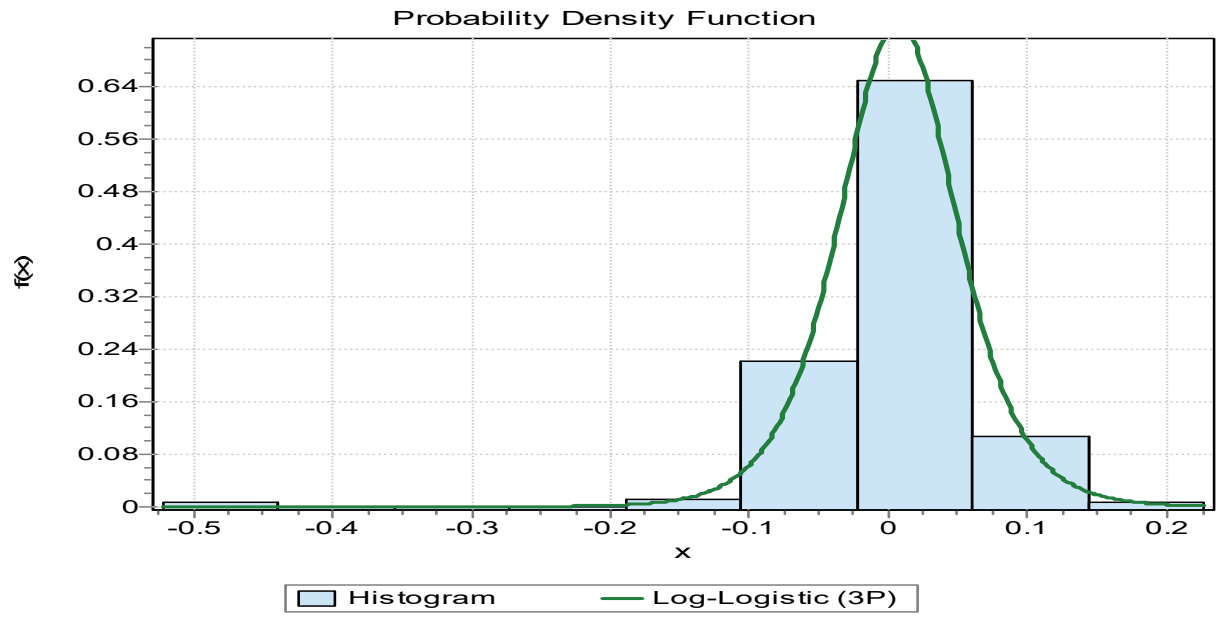

Name of Probability Kolmogorov Distribution Smirnov Rank \begin{tabular}{|ll|l|l}
$\begin{array}{l}\text { Anderson } \\
\text { Rank }\end{array}$ & Darling & Chi- Squared Rank & Average \\
\hline
\end{tabular} 


\begin{tabular}{|l|l|l|l|l|}
\hline Burr & 3 & 3 & 3 & 3.00 \\
\hline Dagum & 2 & 2 & 4 & 2.67 \\
\hline Log - Logistic(3P) & 1 & 1 & 1 & 1.00 \\
\hline
\end{tabular}

Geometric Mean and Volatility (Standard Deviation) of the Macroeconomic indicators used in the case of Exxon Mobil Corporation are given by:

$\mu_{G E O}(X O M)=0.030 / 2951 \%$

$\sigma_{G E O}(X O M)=0.110236167$

Geometric Mean and Volatility (Standard Deviation) of Exxon Mobil Corporation Stock Return are given by: $\mu_{\text {STOCK }}(X U M)=0.0048 / 448$

\section{Results of Stressed SIGMOID and Probit MODELS for Exxon Mobil Corporation:}

Under Exxon Mobil, on the month of October, 2014, the probability of default using the existing Sigmoid is $0.499779809 \%$ and that of probit is $0.500351375 \%$, whereas, using the Stressed Sigmoid and Probit models are: $0.499996206 \%, \quad 0.499994665 \%, \quad 0.500002424 \%, \quad 0.4999988447 \%, \quad 0.499780578 \%, \quad 0.499779039 \%$, $0.499786791 \%, \quad 0.499772826 \%, \quad 0.5015668886 \%, \quad 0.501573051 \%, \quad 0.501542014 \%, \quad 0.501597925 \%$, $0.500348293 \%, 0.500354456 \%, 0.500323419 \%$, and $0.500379339 \%$ respectively. This can be seen below:

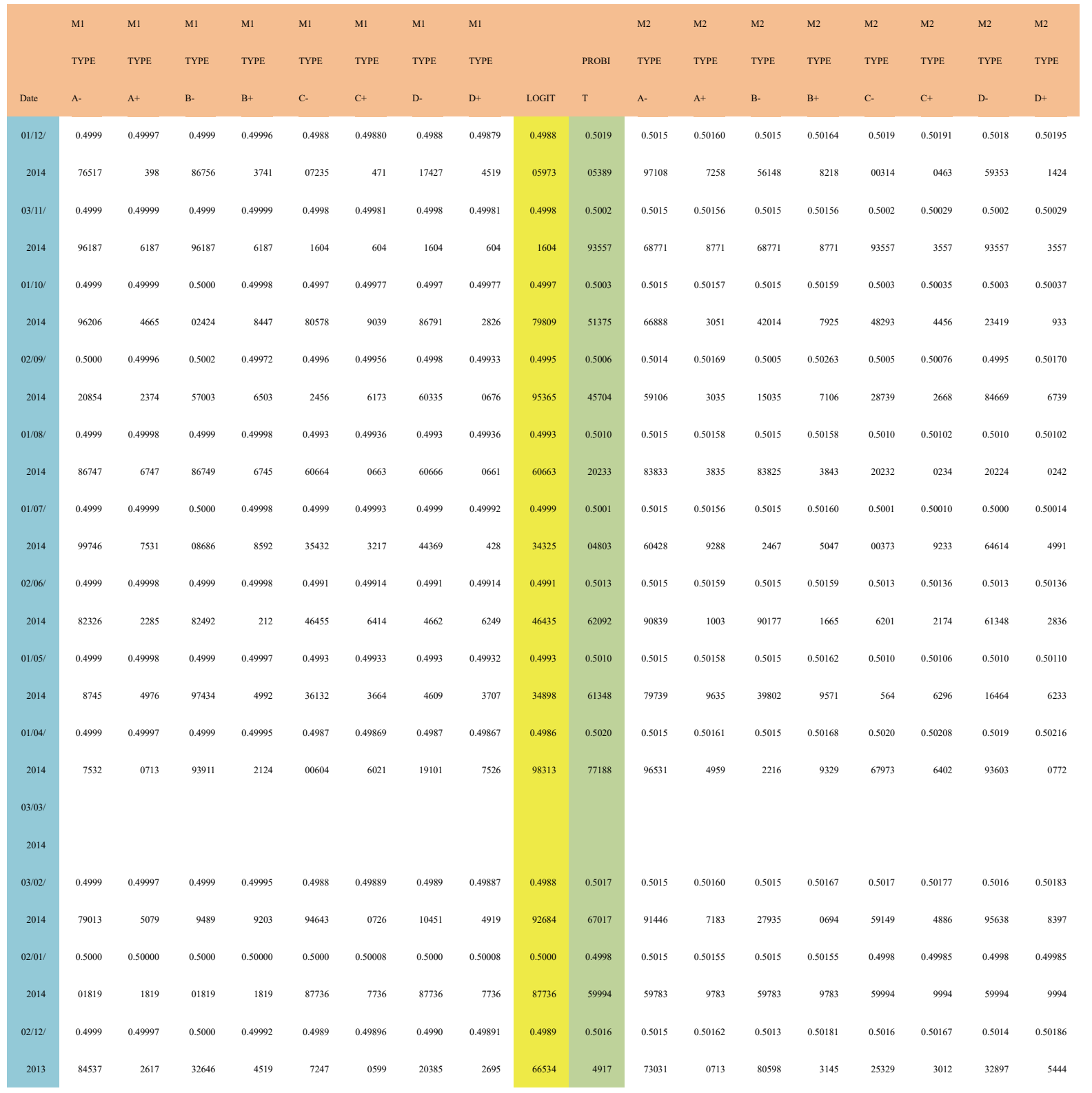




\begin{tabular}{|c|c|c|c|c|c|c|c|c|c|c|c|c|c|c|c|c|c|c|}
\hline $01 / 11 /$ & 0.4999 & 0.49998 & 0.4999 & 0.49996 & 0.4991 & 0.49919 & 0.4992 & 0.49917 & 0.4991 & 0.5012 & 0.5015 & 0.50159 & 0.5015 & 0.50165 & 0.5012 & 0.50129 & 0.5012 & 0.50135 \\
\hline 2013 & 85144 & 1468 & 99982 & 6631 & 96509 & 2845 & 113 & 8055 & 94677 & 85108 & 81972 & 6679 & 22619 & 6031 & 77755 & 2462 & 18403 & 1814 \\
\hline $01 / 10 /$ & 0.4999 & 0.49999 & 0.5000 & 0.49998 & 0.4998 & 0.49981 & 0.4998 & 0.49980 & 0.4998 & 0.5002 & 0.5015 & 0.50157 & 0.5015 & 0.50163 & 0.5002 & 0.50029 & 0.5002 & 0.50034 \\
\hline 2013 & 98017 & 4581 & 11883 & 0716 & 23179 & 9745 & 37035 & 589 & 21462 & 84905 & 6172 & 5463 & 06257 & 0926 & 78034 & 1777 & 22571 & 7239 \\
\hline 03/09/ & 0.4999 & 0.49998 & 0.4999 & 0.49997 & 0.4993 & 0.49929 & 0.4993 & 0.49929 & 0.4992 & 0.5011 & 0.5015 & 0.50158 & 0.5015 & 0.50161 & 0.5011 & 0.50112 & 0.5010 & 0.50114 \\
\hline 2013 & 86147 & 4814 & 91527 & 9435 & 00249 & 892 & 05614 & 3555 & 99584 & 17701 & 83189 & 8521 & 61669 & 0041 & 15035 & 0367 & 93515 & 1887 \\
\hline 01/08/ & 0.4999 & 0.49998 & 0.4999 & 0.49998 & 0.4993 & 0.49939 & 0.4993 & 0.49939 & 0.4993 & 0.5009 & 0.5015 & 0.50158 & 0.5015 & 0.50158 & 0.5009 & 0.50097 & 0.5009 & 0.50097 \\
\hline 2013 & 87397 & 7397 & 87397 & 7397 & 92013 & 2013 & 92013 & 2013 & 92013 & 70206 & 82797 & 2797 & 82797 & 2797 & 70206 & 0206 & 70206 & 0206 \\
\hline
\end{tabular}

Source: Jamilu Auwalu Adamu (2015), Asian Journal of Management Sciences, 03(08), 2015, 10-18

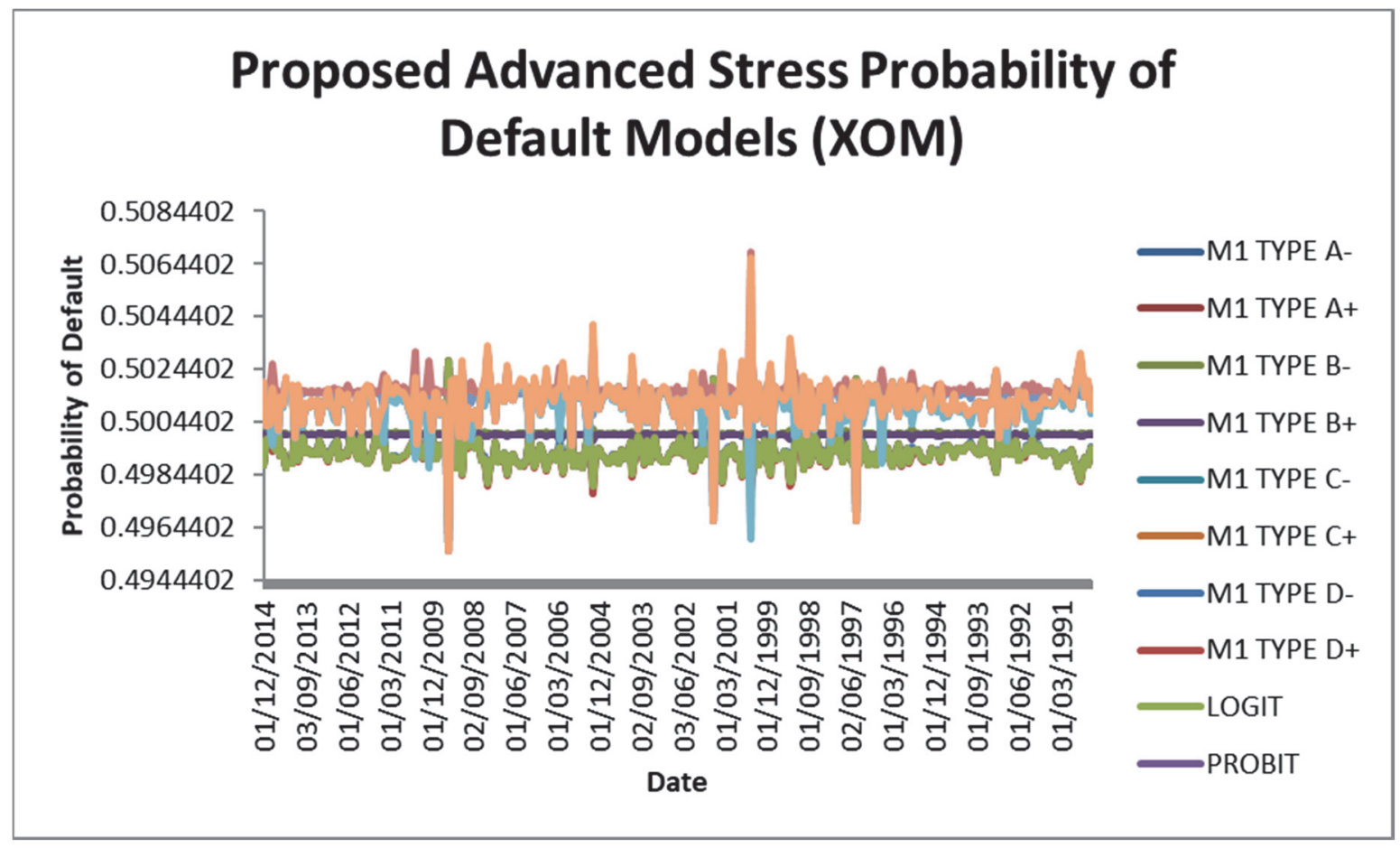

SUMMARY RESULT OF TEST OF GOODNESS OF FIT OF GENERAL ELECTRIC CORPORATION STOCK RETURN 


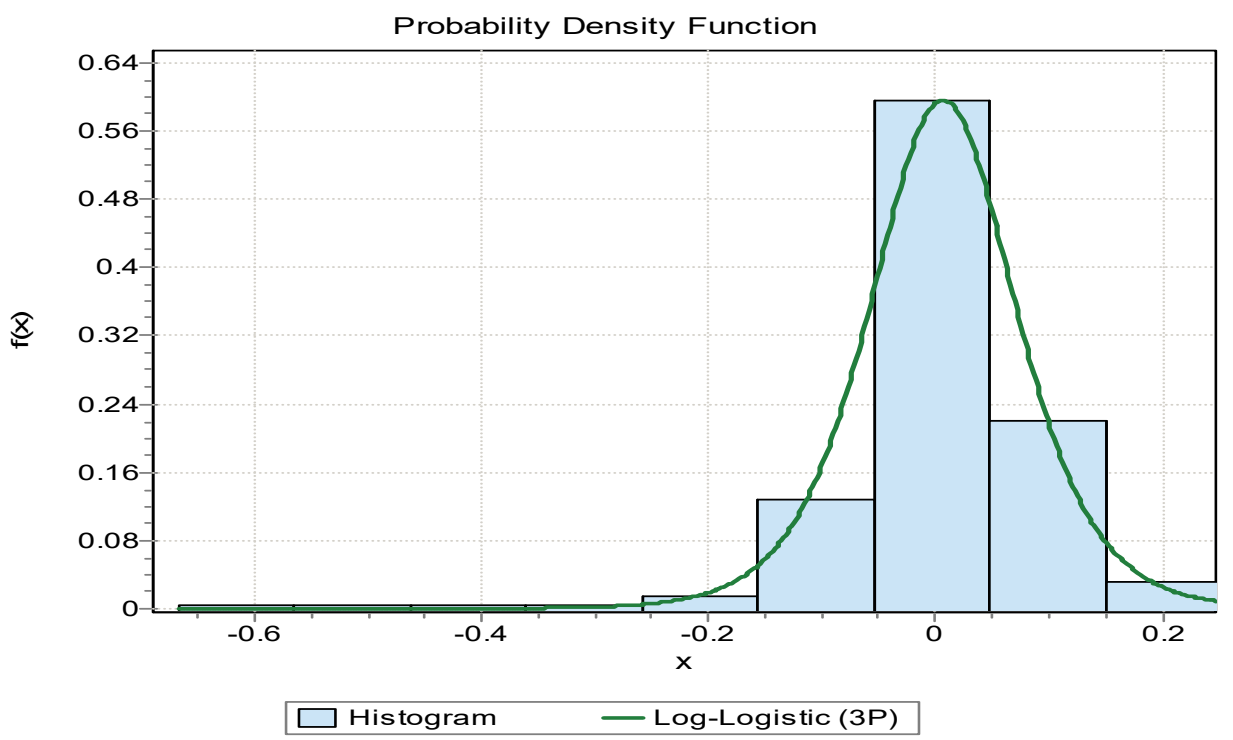

\begin{tabular}{|l|l|l|l|l|}
\hline $\begin{array}{l}\text { Name of Probability } \\
\text { Distribution }\end{array}$ & $\begin{array}{l}\text { Kolmogorov } \\
\text { Smirnov Rank }\end{array}$ & $\begin{array}{l}\text { Anderson Darling } \\
\text { Rank }\end{array}$ & Chi- Squared Rank & Average \\
\hline Error & 3 & 3 & 2 & 2.67 \\
\hline Laplace & 2 & 2 & 3 & 2.33 \\
\hline Log - Logistic (3P) & 1 & 1 & 1 & 1.0 \\
\hline
\end{tabular}

Results of Stressed SIGMOID and Probit MODELS for General Electric:

Under General Electric, on the month of September, 2014, the probability of default using the existing Sigmoid is $0.499256894 \%$ and that of probit is $0.501185825 \%$, whereas, using the Stressed Sigmoid and Probit models are: $0.499973123 \%, 0.499971787 \%, 0.499979127 \%, 0.499965783 \%, 0.49925736 \%, 0.499256228 \%, 0.499263546 \%$, $0.499250241 \%, 0.501608698 \%, 0.501614042 \%, 0.50158468 \%, 0.50163806 \%, 0.501183154 \%, 0.501188497 \%$, $0.501159135 \%$, and $0.501212515 \%$ respectively. All the SIXTEEN (16) proposed banking and economic advanced stressed probability of default models provides good numerical approximations at the time of financial crisis and of course incorporate crisis components as shown in the table below:

\begin{tabular}{|c|c|c|c|c|c|c|c|c|c|c|c|c|c|c|c|c|c|c|}
\hline & M1 & M1 & M1 & M1 & M1 & M1 & M1 & M1 & & & M2 & M2 & M2 & M2 & M2 & M2 & M2 & M2 \\
\hline & TYPE & TYPE & TYPE & TYPE & TYPE & TYPE & TYPE & TYPE & & PROBI & TYPE & TYPE & TYPE & TYPE & TYPE & TYPE & TYPE & TYPE \\
\hline Date & A- & $\mathrm{A}^{+}$ & B- & $\mathrm{B}^{+}$ & c- & $\mathrm{C}+$ & D- & D+ & LOGIT & $\mathrm{T}$ & A- & $\mathrm{A}^{+}$ & B- & $\mathrm{B}^{+}$ & C. & $\mathrm{C}+$ & D- & D+ \\
\hline $01 / 12 /$ & 0.4999 & 0.49995 & 0.4999 & 0.49993 & 0.4987 & 0.49877 & 0.4987 & 0.49875 & 0.4987 & 0.5019 & 0.5015 & 0.50159 & 0.5014 & 0.50166 & 0.5019 & 0.50195 & 0.5018 & 0.50203 \\
\hline 2014 & 56807 & 2572 & 75846 & 3535 & 79722 & 5507 & 98671 & 6559 & 77614 & 50642 & 74875 & 182 & 98709 & 7986 & 42169 & 9114 & 66003 & 528 \\
\hline $03 / 11 /$ & 0.4999 & 0.49997 & 0.4999 & 0.49996 & 0.4994 & 0.49944 & 0.4994 & 0.49943 & 0.4994 & 0.5008 & 0.5016 & 0.50162 & 0.5015 & 0.50166 & 0.5008 & 0.50089 & 0.5008 & 0.50093 \\
\hline 2014 & 80508 & 8257 & 90626 & 8139 & 4489 & 2644 & 54987 & 2548 & 43767 & 87619 & 16732 & 5737 & 76257 & 6212 & 83116 & 2121 & 42641 & 2597 \\
\hline $01 / 10 /$ & 0.4999 & 0.49999 & 0.4999 & 0.49999 & 0.4998 & 0.49989 & 0.4998 & 0.49989 & 0.4998 & 0.5001 & 0.5016 & 0.50164 & 0.5016 & 0.50165 & 0.5001 & 0.50017 & 0.5001 & 0.50017 \\
\hline 2014 & 96113 & 5992 & 96656 & 5448 & 93555 & 3434 & 94098 & 289 & 93494 & 69959 & 48154 & 8638 & 45979 & 0813 & 69717 & 0201 & 67541 & 2376 \\
\hline $02 / 09 /$ & 0.4999 & 0.49997 & 0.4999 & 0.49996 & 0.4992 & 0.49925 & 0.4992 & 0.49925 & 0.4992 & 0.5011 & 0.5016 & 0.50161 & 0.5015 & 0.50163 & 0.5011 & 0.50118 & 0.5011 & 0.50121 \\
\hline 2014 & 73123 & 1787 & 79127 & 5783 & 5756 & 6228 & 63546 & 0241 & 56894 & 85825 & 08698 & 4042 & 8468 & 806 & 83154 & 8497 & 59135 & 2515 \\
\hline $01 / 08 /$ & 0.4999 & 0.49997 & 0.4999 & 0.49995 & 0.4993 & 0.49934 & 0.4993 & 0.49933 & 0.4993 & 0.5010 & 0.5016 & 0.50162 & 0.5015 & 0.50168 & 0.5010 & 0.50104 & 0.5009 & 0.50110 \\
\hline 2014 & 77602 & 4093 & 93377 & 8319 & 50157 & 6656 & 65892 & 0922 & 48407 & 39792 & 08378 & 2416 & 45274 & 552 & 32773 & 6811 & 69669 & 9915 \\
\hline $01 / 07 /$ & 0.5000 & 0.49998 & 0.5000 & 0.49992 & 0.4998 & 0.49982 & 0.4998 & 0.49975 & 0.4998 & 0.5002 & 0.5016 & 0.50167 & 0.5013 & 0.50193 & 0.5002 & 0.50030 & 0.4999 & 0.50056 \\
\hline 2014 & 00848 & 6356 & 65999 & 1226 & 34629 & 0147 & 99736 & 506 & 27388 & 7545 & 16993 & 4961 & 56423 & 553 & 46466 & 4434 & 85896 & 5003 \\
\hline $02 / 06 /$ & 0.4999 & 0.49996 & 0.4999 & 0.49995 & 0.4990 & 0.49909 & 0.4991 & 0.49908 & 0.4990 & 0.5014 & 0.5015 & 0.50160 & 0.5015 & 0.50165 & 0.5014 & 0.50144 & 0.5013 & 0.50148 \\
\hline
\end{tabular}




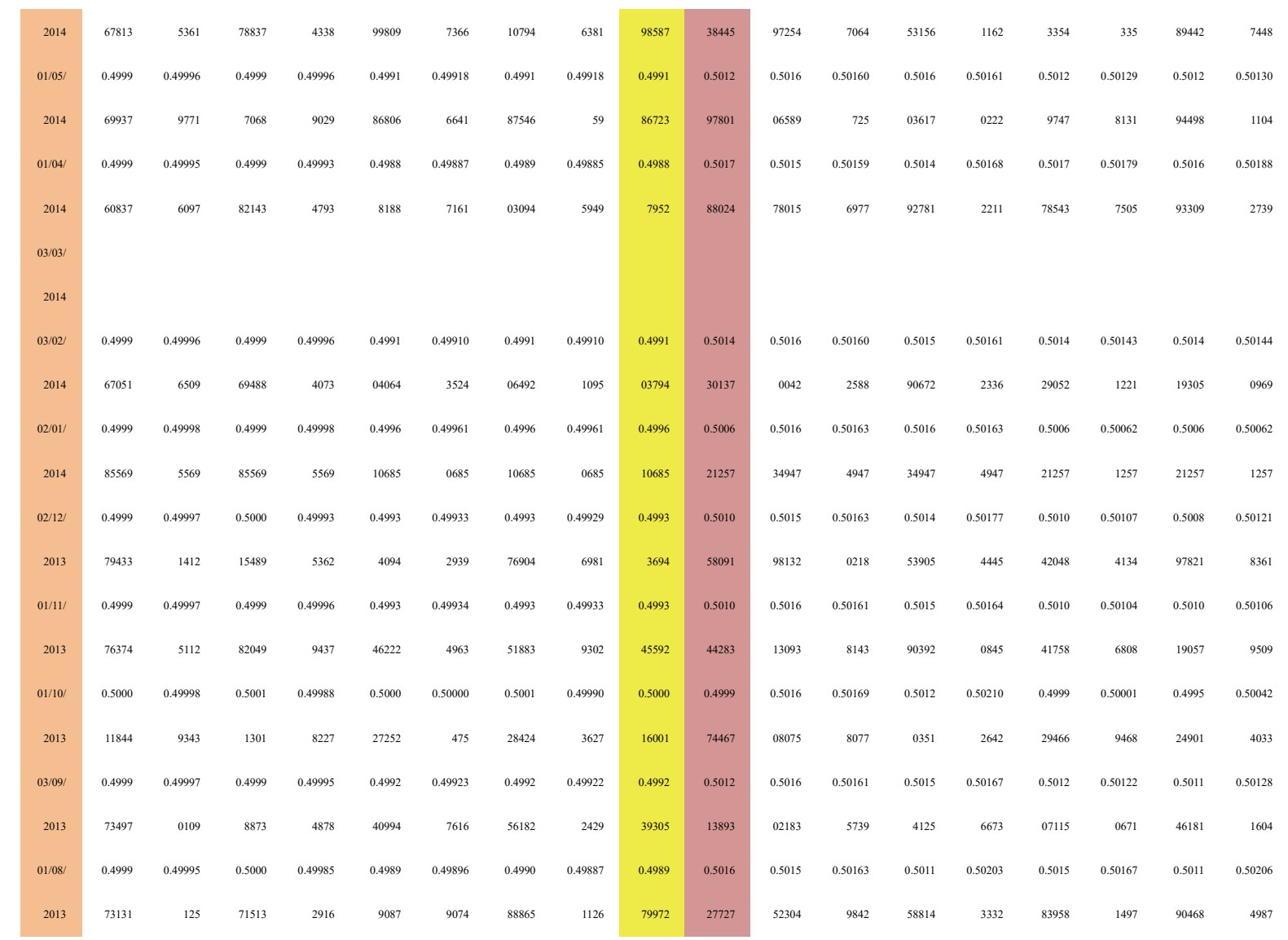

Source: Jamilu Auwalu Adamu (2015), Asian Journal of Management Sciences, 03(08), 2015, 10-18

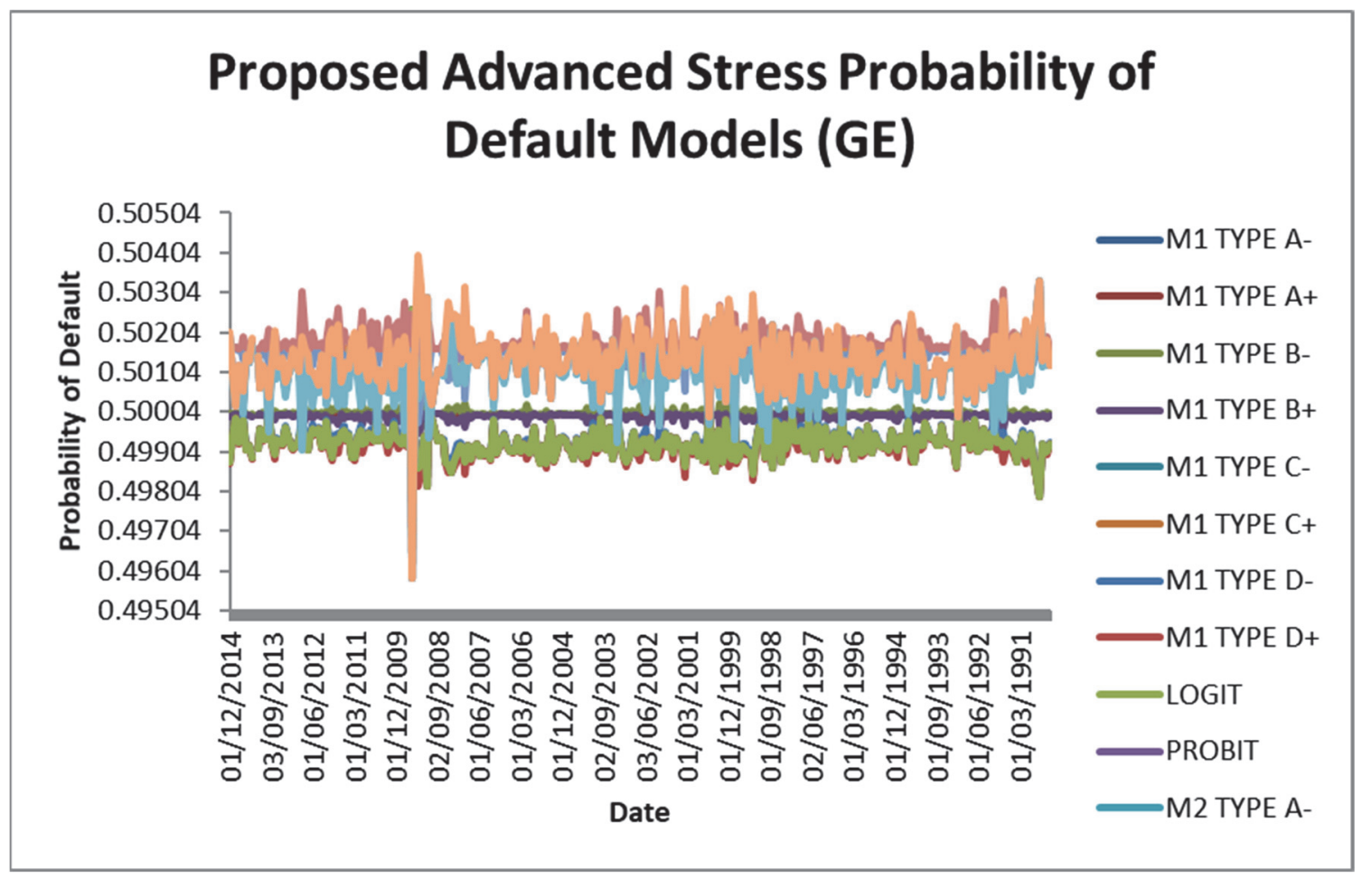




\section{Appendix C}

\section{Advanced Optimized Activation Functions}

The Ranking of the Advanced Optimized Activation Functions was obtained using Jameel's Criterion (second version). The author considered Eleven (11) out of Fifty (50) World's Biggest Public Companies by FORBES as of 2015 Ranking Regardless of the platform in which they are listed, Time Series (Short or Long Terms), Old or Recently Listed Companies. This can be seen below:

\begin{tabular}{|l|l|l|}
\hline $\begin{array}{l}\text { Advanced Optimized Activation } \\
\text { Functions }\end{array}$ & $\begin{array}{l}\text { Frequency regardless of the Time } \\
\text { Series }\end{array}$ & Order of Hierarchy \\
\hline Log-Logistic (3P) & 50 & $1^{\text {st }}$ \\
\hline Cauchy & 33 & $2^{\text {nd }}$ \\
\hline Pearson 5 (3P) & 25 & $3^{\text {rd }}$ \\
\hline Burr (4P) & 19 & $4^{\text {th }}$ \\
\hline Fatique Life (3P) & 12 & $5^{\text {th }}$ \\
\hline Inv. Gaussian (3P) & 10 & $6^{\text {th }}$ \\
\hline Dagum (4P) & 9 & $7^{\text {th }}$ \\
\hline Lognormal (3P) & 8 & $8^{\text {th }}$ \\
\hline $\begin{array}{l}\text { Normal, Pearson 6 (4P), Gen. Gamma } \\
\text { (4P), Error, Laplace, Hypersecant, } \\
\text { Gamma, Johnson SU, Beta, and } \\
\text { Uniform }\end{array}$ & Frequency less than or equal to 8 & $9^{\text {th }}$ and below it \\
\hline
\end{tabular}

Source: Jamilu Auwalu Adamu (2015), Asian Journal of Management Sciences, 03(12), 2015, 01-07

\section{Copyrights}

Copyright for this article is retained by the author(s), with first publication rights granted to the journal.

This is an open-access article distributed under the terms and conditions of the Creative Commons Attribution license (http://creativecommons.org/licenses/by/4.0/). 